\title{
Influence of Annular Dynamics and Material Behavior in Finite Element Analysis of Barlow's Mitral Valve Disease
}

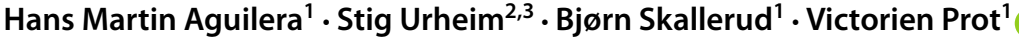

Received: 14 October 2020 / Accepted: 30 March 2021 / Published online: 21 May 2021

(c) The Author(s) 2021

\begin{abstract}
Barlow's disease affects the entire mitral valve apparatus, by altering several of the fundamental mechanisms in the mitral valve which ensures unidirectional blood flow between the left atrium and the left ventricle. In this paper, a finite element model of a patient diagnosed with Barlow's disease with patient-specific geometry and boundary conditions is presented. The geometry and boundary conditions are extracted from the echocardiographic assessment of the patient prior to surgery. Material properties representing myxomatous, healthy human and animal mitral valves are implemented and computed response are compared with each other and the echocardiographic images of the patient. This study shows that the annular dilation observed in Barlow's patients controls several aspects of the mitral valve behavior during ventricular systole. The coaptation of the leaflets is observed to be highly dependent on annular dilation, and the coaptation area reduces rapidly at the onset of mitral regurgitation. Furthermore, the leaflet material implementation is important to predict lack of closure in the FE model correctly. It was observed that using healthy human material parameters in the Barlow's diseased FE geometry gave severe lack of closure from the onset of mitral regurgitation, while myxomatous material properties showed a more physiological leakage.
\end{abstract}

Mathematics Subject Classification 74L15 · 92C10

Keywords Finite element modeling · Barlow's disease $\cdot$ Mitral valve $\cdot$ Patient-specific · Echocardiography

\section{Introduction}

The mitral valve apparatus is a complex structure that ensures one-way blood flow between the left atrium and left ventricle. The valvular structure consists of the anterior and posterior leaflets, the annulus, the papillary muscles and the chordae tendinae. These components

V. Prot

victorien.prot@ntnu.no

1 Department of Structural Engineering, Norwegian University of Science and Technology, Trondheim, Norway

2 Department of Heart Disease, Haukeland University Hospital, Bergen, Norway

3 Clinical Institute 2, University of Bergen, Bergen, Norway 
must work in concert to ensure proper valve closure, preventing blood from regurgitating during ventricular systole.

Mitral regurgitation is the second most common valvular heart disease in European countries, where degenerative diseases such as Fibroelastic deficiency and Barlow's Disease (BD) are the most frequent etiology [1]. BD affects the entire mitral valve apparatus leading to billowing and late systolic prolapse of diseased segments. BD is further characterized by myxomatous thickening of the mitral valve leaflets and chordae together with severe annular dilation and a diminished annular saddle shape, often accompanied by mitral annular disjunction (MAD) [2-5].

In a study performed by Fornes et al. [6], it was discovered that among all degenerative mitral valve diseases, the myxomatous infiltration of the leaflets were most severe in BD patients. For myxoid leaflets, accumulation of glycosaminoglycans in the spongiosa layer causes the leaflets to swell and thicken. Myxoid chordae has an increased cross-sectional area due to accumulation of fibrous tissue around its collagen core [7]. In addition to the vacuolization of the spongiosa layer, the leaflet structure has a disrupted connective tissue architecture [8-11]. The collagen fibrils are disorganized with varying length and thickness, with a collagen density appearing diminished due to the invasion of the spongiosa into the fibrosa layer [9]. Furthermore, the elastin is fragmented and granulated often appearing in clusters $[2,4,9,12]$. Lastly, Barber et al. [4, 7] studied the mechanical properties of myxomatous leaflet tissue and chordae, by means of uniaxial tensile tests. They reported that the myxomatous mitral valve leaflets were more extensible and less stiff compared to normal mitral valve leaflets, both in the radial and circumferential direction. Moreover, the myxomatous chordae were shown to be altered most in terms of failure load, failing at loads one-half of those of normal chordae.

Creating patient-specific finite element models able to reproduce the patient's in vivo behavior, could potentially improve surgical planning and procedures as well as enable the development of new and sophisticated surgical implants. Since the pioneering work of Kunzelman et al. [13], various finite element models of the mitral valve have been published, continuously evolving in complexity. Several finite element studies have focused on the implementation of constitutive models in their numerical simulations [13-18], creating the basis for further improvement of the finite element models. Especially in recent years, the studies have focused on the structural effects of mitral valve repair by implementing mitral clips, annuloplasty rings, artificial chordae and performing papillary muscle relocation in their FE models [18-23]. Our goal is to establish FE models of mitral valves diagnosed with $\mathrm{BD}$ that can simulate and improve the understanding of this disease state. In the future, these models may be used to improve surgical planning.

To our knowledge, only two studies have reported the use of myxomatous leaflet properties in their FE-models [17, 24]. In both studies, the mechanical response of myxomatous tissue has been acquired by fitting constitutive models to the experimental data presented by Barber et al. [4]. In the paper by Dal Pan et al. [17], the experimental data were fitted to the isotropic reduced polynomial strain-energy function available in the Abaqus material library [25]. In the study, the "edge-to-edge" technique was applied to a finite element model of the mitral valve [17]. However, the paper did not include any material parameters for the presented constitutive model. In the paper by Zhong et al. [24], the constitutive model that was used to fit the experimental data in Barber et al. [4] assumes a two layered composite, each layer being reinforced by one family of fiber orthogonal with respect to each other. In the present paper the myxomatous leaflet properties described by Barber et al. [4] are fitted to the constitutive Gasser-Ogden-Holzapfel (GOH) model available in the Abaqus material library [25], accounting for the mean leaflet thickness observed in BD patients [2]. 
The mitral leaflets are known to be reinforced by only one collagen fiber family [26, 27], therefore we employed only one family of fiber in the GOH-model. To our knowledge, no study has combined myxomatous mitral valve leaflet and chordae material behavior with a patient-specific mitral valve geometry and boundary conditions on a mitral valve diagnosed with BD.

In this paper, a patient-specific mitral valve model of a patient diagnosed with BD is studied using the commercial solver ABAQUS Explicit 2019 (SIMULIA, Dassault Systémes). The finite element model is constructed by extracting the mitral valve leaflet geometry from 3D echocardiographic data of the patient, where also patient-specific annular and papillary muscle movement are extracted and used as dynamic boundary conditions. The mitral valve is created using an image modality with high frame rate (3D echocardiography) because of the severe alterations of the mitral annulus during systole observed in BD patients $[2,3,28]$. Due to its temporal-resolution echocardiography is an imaging technique well suited to account for this dynamic behavior. Material properties representing myxomatous, healthy human and animal material parameters are further implemented in the FE model. The global responses are compared with echocardiography and the coaptation area is calculated for each individual analysis.

The paper is organized as follows. First the continuum mechanical framework and the material models used together with the acquired material parameters from fitting are presented. Then the FE geometry and boundary conditions are described. Finally, the results are presented and discussed.

\section{Methods}

\subsection{Continuum Mechanical Framework and Constitutive Models}

\subsection{Kinematics}

We consider a deformable body in two different instantaneous configurations $\Omega_{0}$ and $\Omega$, representing the reference and current configuration, respectively. A particle in the reference configuration $\Omega_{0}$ is defined by the position vector $\mathbf{X}$. The position of the same particle in the current configuration $\Omega$ is further defined by the vector $\mathbf{x}$. The relationship between the two configurations is described by the deformation map relationship $\mathbf{x}=\mathbf{x}(\mathbf{X}, t)$. The deformation gradient $\mathbf{F}$ is defined as

$$
\mathbf{F}=\frac{\partial \mathbf{x}}{\partial \mathbf{X}}
$$

The volume ratio is defined as $J=\operatorname{det} \mathbf{F}$, where $J=1$ describes an isochoric transformation. Furthermore, the right and left Cauchy-Green tensors are defined as $\mathbf{C}=\mathbf{F}^{\mathbf{T}} \mathbf{F}$ and $\mathbf{b}=\mathbf{F F}^{\mathbf{T}}$ respectively. Moreover, the distortional part of the right and left Cauchy-Green can be written as $\overline{\mathbf{C}}=J^{-\frac{2}{3}} \mathbf{F}^{\mathbf{T}} \mathbf{F}$ and $\overline{\mathbf{b}}=J^{-\frac{2}{3}} \mathbf{F F}^{\mathbf{T}}$.

For an anisotropic material reinforced by a family of fibers, the mean fiber direction in the reference configuration is defined by the unit vector $\mathbf{a}_{0}$. The mapping of the fiber direction from the reference configuration to the current configuration is expressed as $\mathbf{a}=\mathbf{F a}_{0}$. 


\subsection{Strain-Energy Function and Stress Tensors}

For hyperelastic materials the strain-energy function (SEF) $\Psi$ is introduced in order to describe the response of the material. The SEF $\Psi$ for incompressible hyperelastic transversely isotropic materials can be expressed in terms of five invariants, $I_{1}, I_{2}, J, I_{4}, I_{5}$ [29] as,

$$
\Psi=\tilde{\Psi}\left(I_{1}, I_{2}, I_{4}, I_{5}\right)+p(1-J),
$$

where the principal invariants of $\mathbf{C}$ (i.e., $I_{1}, I_{2}, J$ ) are related to isotropic elasticity and defined as,

$$
I_{1}=\operatorname{tr}(\mathbf{C}), \quad I_{2}=\frac{1}{2}\left[I_{1}^{2}-\operatorname{tr}\left(\mathbf{C}^{2}\right)\right], \quad J=\sqrt{\operatorname{det}(\mathbf{C})} .
$$

For an incompressible material $J=1$. The invariants $I_{4}, I_{5}$ serve to describe the transversely isotropic properties of the material, expressed by the fiber direction in the reference configuration $\mathbf{a}_{0}$ and the right Cauchy-Green tensor $\mathbf{C}$,

$$
I_{4}=\mathbf{a}_{0} \cdot \mathbf{C} \mathbf{a}_{0}, \quad I_{5}=\mathbf{a}_{0} \cdot \mathbf{C}^{2} \mathbf{a}_{0},
$$

and $p$ is the Lagrange multiplier. The second Piola-Kirchhoff stress tensor $\mathbf{S}$ can be derived from equation (2) giving,

$$
\mathbf{S}=2 \sum_{\substack{i=1 \\ i \neq 3}}^{5} \frac{\partial \Psi}{\partial \mathbf{I}_{i}} \frac{\partial \mathbf{I}_{i}}{\partial \mathbf{C}}+p \mathbf{C}^{-1},
$$

where the scalar $p$ can be determined from the plane stress condition. In this work, we apply this constitutive model to mitral leaflets that may be considered as thin sheets, thus assuming that the normal stress in the out of plane direction (denoted 3-direction) $S_{33}$ is zero, which leads to:

$$
p=-2 \sum_{\substack{i=1 \\ i \neq 3}}^{5} \frac{\partial \Psi}{\partial \mathbf{I}_{i}} \frac{\partial \mathbf{I}_{i}}{\partial \mathbf{C}} C_{33} .
$$

Lastly the second Piola-Kirchhoff stress tensor $\mathbf{S}$ can be transformed to the Cauchy stress tensor $\sigma=\frac{1}{J} \mathbf{F S F}^{\mathbf{T}}$ by the push-forward operation of $\mathbf{S}$ [14]. This was used for material parameter fitting presented in Sect. 2.4.

\subsection{Material Models and Parameters}

The constitutive model used to analyze the response of the mitral leaflets, is a hyperelastic anisotropic material model named Gasser-Ogden-Holzapfel $(\mathrm{GOH})$, which is available through the material library in Abaqus [25]. The material model was originally developed to model the mechanical response of arterial layers with a distributed collagen fiber orientation $[30,31]$. The SEF W is defined in terms of the deviatoric strain invariants $\bar{I}_{1}$ and $\bar{I}_{4}$, which are defined as,

$$
\bar{I}_{1}=\operatorname{tr} \overline{\mathbf{C}}, \quad \bar{I}_{4}=\mathbf{a}_{0} \cdot \overline{\mathbf{C}} \cdot \mathbf{a}_{0} .
$$


Thus,

$$
W\left(\bar{I}_{1}, \bar{I}_{4}, J\right)=C_{10}\left(\bar{I}_{1}-3\right)+\frac{1}{D}\left(\frac{J^{2}-1}{2}-\ln (J)\right)+\frac{k_{1}}{2 k_{2}}\left(\exp \left\{k_{2}(\bar{E})^{2}\right\}-1\right),
$$

with,

$$
\bar{E}=\kappa\left(\bar{I}_{1}-3\right)+(1-3 \kappa)\left(\bar{I}_{4}-1\right),
$$

where $C_{10}, \mathrm{D}, k_{1}, k_{2}$ and $\kappa$ are material parameters. D is a material constant that controls the compressibility. Due to the high fluid content in soft tissues such as mitral valve tissue, the tissue is assumed to be nearly incompressible [32]. Note that when D is set to zero, the ABAQUS Explicit solver computes a default value of D that ensures a nearly incompressible response of the material model. The dispersion parameter $\kappa$ describes the distribution of the fibers. When $\kappa=0$, there is no dispersion of the fibers, while $\kappa=\frac{1}{3}$ describes an isotropic material where the fibers are uniformly distributed [25].

\subsubsection{Material Parameter Fitting}

In order to describe the mechanical behavior of the mitral valve apparatus, the material parameters of myxomatous, human and animal leaflet and chordae were of particular interest to implement in the finite element model.

In the papers published by Barber et al. [4, 7] the mechanical response of myxomatous leaflet tissue and chordae were described. The myxomatous tissue properties were acquired by resecting circumferential and radial strips mainly from the posterior leaflet and performing uniaxial tensile tests on the strips [4]. The radial direction is defined as the direction from the free edge to the annulus, while the circumferential direction is parallel to the annulus. Moreover, the mechanical properties presented in the papers are in terms of extensibility, stiffness, failure strain and failure strength, but no constitutive model was suggested. In the current study, the corresponding material parameters for the GOH-model describing myxomatous tissue were obtained by an inverse FE method, where the goal was to reproduce the extensibility and stiffness measure given in $[4,7]$.

The inverse FE procedure was done by creating a uniaxial test in Abaqus, where the width and length of the specimen was set to 10 and $14 \mathrm{~mm}$ as described in Barber et al. [4]. One end of the specimen was fixed, while the other end was subjected to a load equivalent of the failure strength given in [4] and constrained in the lateral direction. From the Tension(N/m) vs strain response, extensibility and stiffness were computed and fitted to the data reported in Barber et al. [4]. As the data was given in membrane tension and the thickness of the samples is unknown. The fitting was done for a thickness of $3 \mathrm{~mm}$, which is the mean thickness of Barlow leaflets as reported by [2]. Note that for the FE analyses, the thickness of the mitral leaflets was set to $3 \mathrm{~mm}$, in order to correctly model the same tension-strain relationship as reported in [4]. The response curve is plotted in Fig. 1, while the obtained material parameters are presented in Table 1.

Little is known regarding the anterior leaflet of myxomatous mitral valves, due to leaflets specimens acquired for material testing are primarily resected from the posterior leaflet during surgery [4]. Consequently, both leaflets are modeled with the same material parameters for myxomatous tissue.

The healthy human material parameters were acquired by fitting the response of the stress-stretch relationships predicted by the SEF from equation (8) to the SEF $\Psi_{\text {human }}$ with 
Fig. 1 Tension-strain curves in the same format as the mitral valve leaflet response presented by Barber [4]

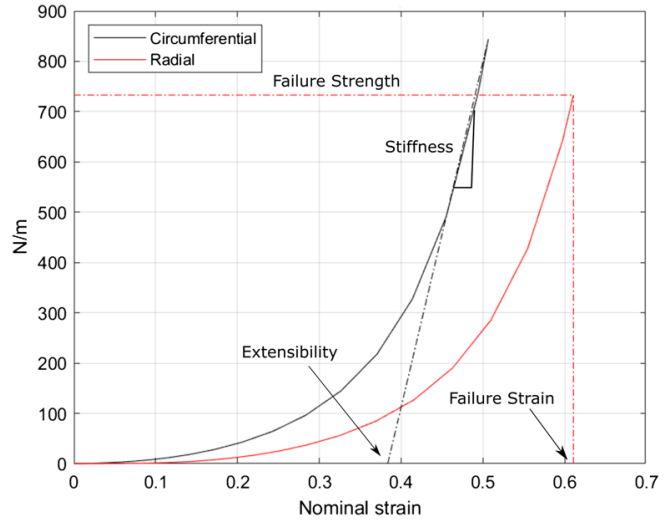

corresponding material parameters reported in [16]. The SEF $\Psi_{\text {human }}$ can be expressed as

$$
\begin{aligned}
\Psi_{\text {human }}\left(\bar{I}_{1}, \bar{I}_{4 i}\right)= & b_{0}\left\{\exp \left[b_{1}\left(\bar{I}_{1}-3\right)\right]-1\right\} \\
& +\frac{k_{1}}{2 k_{2}} \sum_{i=1}^{2}\left[\exp \left\{k_{2}\left[\kappa\left(\bar{I}_{1}-3\right)+(1-3 \kappa)\left(\bar{I}_{4 i}-1\right)\right]^{2}\right\}-1\right] \\
& +\frac{1}{D}\left(J^{2}-1\right)^{2}, i=1,2,
\end{aligned}
$$

where the parameters $b_{0}$ and $b_{1}$ describe the matrix material [16]. In order to obtain the parameters in the GOH-model, the lsqnonlin function from the Optimization Toolbox of Matlab was used to perform a nonlinear least square fitting. In [16], the healthy human material parameters were obtained using a load-controlled biaxial test, resulting in off-biaxial displacements due to the anisotropic behavior of the tissue specimens. Therefore, GOHparameters were fitted to an off-biaxial test close to the stress-stretch response presented in [16] for each leaflet. In Fig. 2, the curve fitting for the SEF in equation (8) to the SEF (10) is illustrated for both the anterior and posterior leaflet. Furthermore, the obtained material parameters from the curve fitting are reported in Table 1.

May-Newman and Yin [33] proposed a SEF for porcine leaflet tissue with corresponding material parameters. The suggested model in [33] has an exponential form in terms of the invariants $I_{1}$ and $I_{4}$.

$$
\Psi_{\text {porcine }}\left(I_{1}, I_{4}\right)=c_{0}\left[\exp \left\{c_{1}\left(I_{1}-3\right)^{2}+c_{2}\left(\sqrt{I_{4}}-1\right)^{4}\right\}-1\right]+p(J-1),
$$

where $c_{i}, i=0,1,2$, are material parameters, and $p$ is the Lagrange-multiplier.

The lsqnonlin function is also used to fit the GOH-model to the SEF presented by MayNewman and Yin [33], as done previously by our group [14]. In Table 1, the acquired material parameters from the non-linear regression is presented.

Lastly, in Fig. 3 the material fitting is plotted for an equibiaxial and an off-biaxial test, comparing the response of the myxomatous, human and porcine material parameters. When the material models are implemented in Abaqus, the leaflet density is set to $1000 \mathrm{~kg} / \mathrm{m}^{3}$.

\subsubsection{Chordae Parameters}

In this paper, the FE model is analyzed with three different material behaviors for the chordae namely myxomatous, healthy human and ovine (Fig. 4). For both healthy human and 


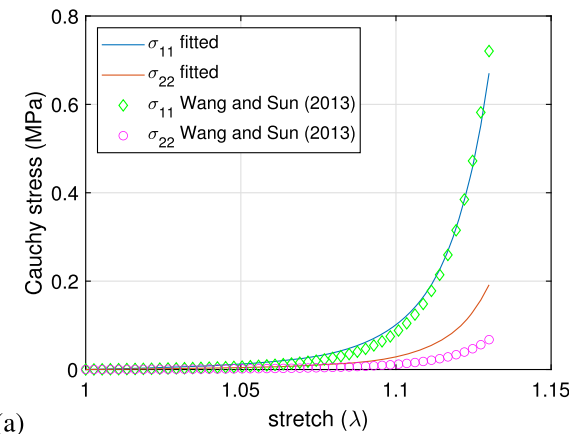

(a)
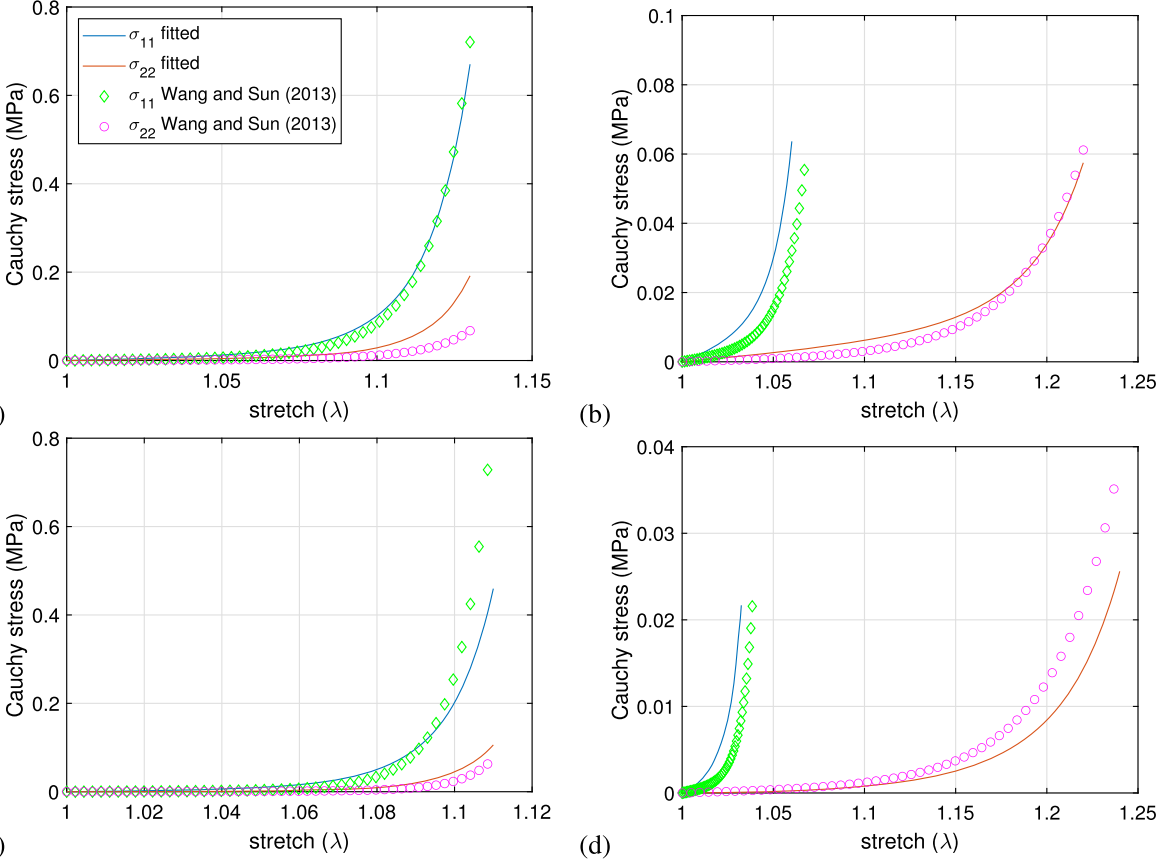

(b)

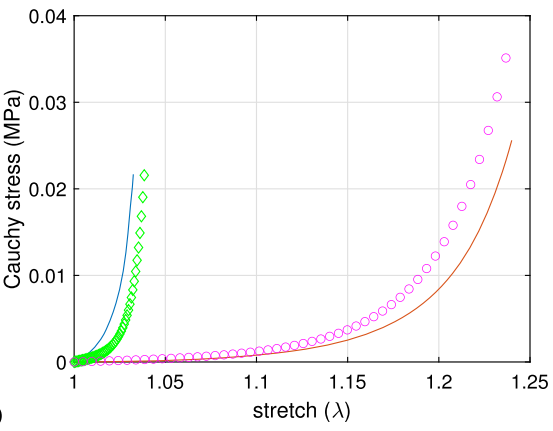

Fig. 2 Cauchy-stress vs stretch curves of nonlinear data-fitting between the SEFs described in equation (8) and equation (10) with corresponding material parameters [16]. (a) Equibiaxial displacement anterior leaflet. (b) 3:1 off-biaxial displacement anterior leaflet. (c) Equibiaxial displacement posterior leaflet. (d) 5:1 offbiaxial displacement posterior leaflet. $\left(\sigma_{11}\right)$ Cauchy-stress in the circumferential direction. $\left(\sigma_{22}\right)$ Cauchystress in the radial direction

Table 1 Material parameters for modeling of human, myxomatous and porcine material response in the GOH-model (8).

(AL)-Anterior Leaflet, (PL)-Posterior Leaflet

\begin{tabular}{lllrl}
\hline & $c_{10}(\mathrm{MPa})$ & $k_{1}(\mathrm{MPa})$ & \multicolumn{1}{l}{$k_{2}$} & $\kappa$ \\
\hline AL Healthy [16] & 0.0074 & 0.189 & 210.3 & 0.230 \\
PL Healthy [16] & 0.0001 & 0.167 & 280.0 & 0.219 \\
AL Porcine [33] & 0.0010 & 0.024 & 50.9 & 0.173 \\
PL Porcine [33] & 0.0010 & 0.021 & 51.8 & 0.267 \\
AL/PL & 0.0010 & 0.453 & 14.7 & 0.316 \\
Myxomatous [4] & & & & \\
\hline
\end{tabular}

ovine, Zuo et al. [34] described the response of anterior marginal (AM), posterior marginal (PM) and anterior strut (AS), using the Ogden SEF with corresponding material parameters. When assumed incompressible, the SEF is,

$$
\Psi_{\text {Ogden }}=\sum_{i=1}^{3} \frac{2 \mu_{i}}{\alpha_{i}^{2}}\left(\lambda_{1}^{\alpha_{i}}+\lambda_{2}^{\alpha_{i}}+\lambda_{3}^{\alpha_{i}}-3\right),
$$

where $\mu_{i}$ and $\alpha_{i}$ are material constants and $\lambda_{j}(j=1,2,3)$ are the principal stretches. In Table 2, the material parameters for the marginal and strut chordae are presented. 


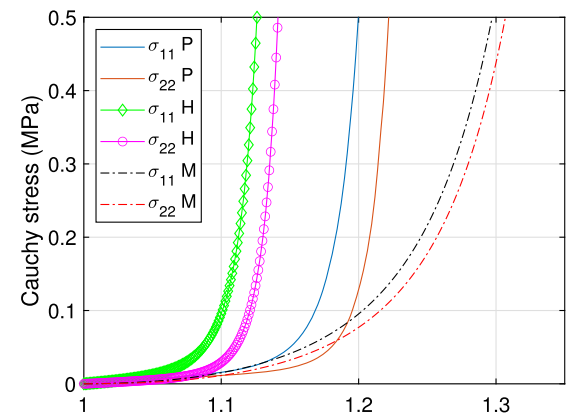

(a)

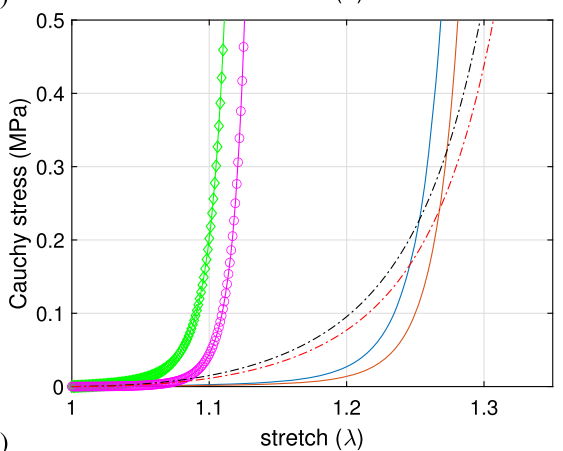

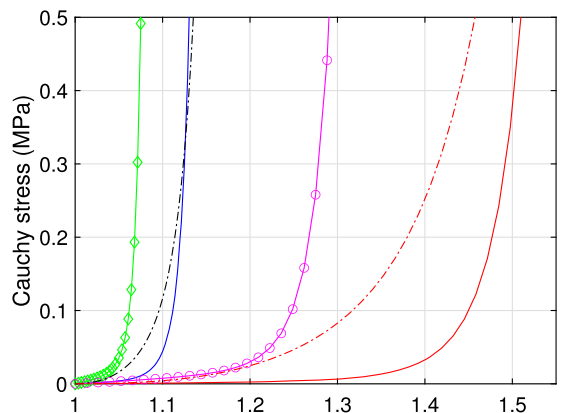

(b)

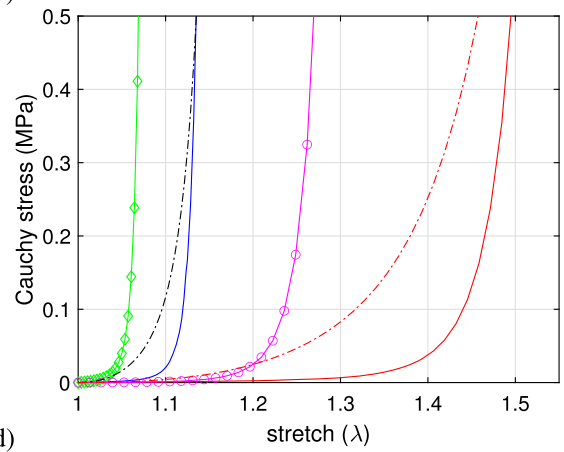

Fig. 3 Cauchy stress vs stretch responses from equibiaxial (a and c) and 5:1 off-biaxial (b and d) tests for Porcine (P), Healthy Human (H), Myxomatous (M) leaflet parameters. (a and b) - responses for anterior leaflet. (c and d) - responses for posterior leaflet. $\left(\sigma_{11}\right)$ Cauchy-stress in the circumferential direction. $\left(\sigma_{22}\right)$ Cauchy-stress in the radial direction

Table 2 Material parameters for healthy and ovine chordae

\begin{tabular}{|c|c|c|c|c|c|c|}
\hline & \multicolumn{3}{|c|}{ Human } & \multicolumn{3}{|l|}{ Ovine } \\
\hline & $\overline{\mathrm{AM}}$ & AS & PM & $\mathrm{AM}$ & AS & PM \\
\hline$\mu_{1}(\mathrm{MPa})$ & 8.91 & 9.61 & 9.57 & 0.37 & 0.85 & 0.66 \\
\hline$\alpha_{1}$ & 27.02 & 30.86 & 22.78 & 11.7 & 28.03 & 29.67 \\
\hline$\mu_{2}(\mathrm{MPa})$ & 12.19 & 7.99 & 10.61 & 1.79 & 0.21 & 1.37 \\
\hline$\alpha_{2}$ & 20.91 & 27.65 & 21.68 & 5 & 5.47 & 18.25 \\
\hline$\mu_{3}(\mathrm{MPa})$ & 12.78 & 7.81 & 10.65 & 0.33 & 0.16 & 1.46 \\
\hline$\alpha_{3}$ & 20.89 & 30 & 21.35 & 34.06 & 25.06 & 19.45 \\
\hline
\end{tabular}

For the myxomatous response, the experimental data from Barber et al. [7] were fitted for a uniaxial test. We first fitted the Ogden model to the experimental data, however the fit obtained by the reduced polynomial SEF of order 5 was substantially better. The reduced polynomial SEF can be written as,

$$
\Psi_{\text {Reduced }}=\sum_{i=1}^{5} C_{i 0}\left(\bar{I}_{1}-3\right)^{i},
$$


Fig. 4 Nominal stress vs nominal strain curves of the anterior Myxomatous (M), Healthy Human $(\mathrm{H})$ and Ovine $(\mathrm{O})$ marginal chordae parameters for

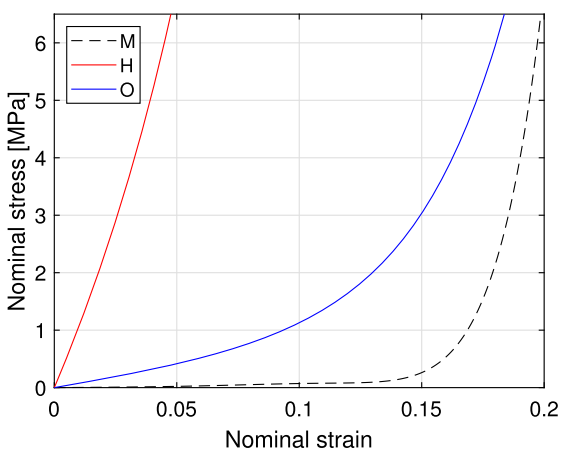

Table 3 Material parameters for myxomatous chordae

\begin{tabular}{llllll}
\hline & $\begin{array}{l}C_{10} \\
(\mathrm{MPa})\end{array}$ & $\begin{array}{l}C_{20} \\
(\mathrm{MPa})\end{array}$ & $\begin{array}{l}C_{30} \\
(\mathrm{MPa})\end{array}$ & $\begin{array}{l}C_{40} \\
(\mathrm{MPa})\end{array}$ & $\begin{array}{l}C_{50} \\
(\mathrm{MPa})\end{array}$ \\
\hline Basal & 0.7694 & 0 & 0 & 0 & 26793 \\
Marginal & 0.05 & 0 & 0 & 0 & 772.8 \\
\hline
\end{tabular}

when assumed incompressible. During the fitting procedure, the material constants had a lower bound of 0 , in order to avoid a non-physical fitting with negative values. This leads to a polynomial fit with a first and a fifth order term. The fitted hyperelastic constants are presented in Table 3. Basal chordae are according to Barber et al. [4], inserted either into the belly of the leaflet or near the annulus, hence the basal chordae fitting is used as strut chordae in the finite element model.

\subsection{Geometry}

The patient-specific geometry was created from 3D echocardiographic data of a patient diagnosed with BD. The recordings were imported into an in-house Matlab GUI which enables manual extraction of geometrical points from echocardiography. At the end-diastolic configuration the annulus, leaflet edges and papillary muscle tips were identified (Fig. 5).

The annulus was created by extracting 12 points along the annular perimeter and performing a cubic spline interpolation as shown in Fig. 5a. The interpolated closed spline was compared against echocardiographic images and showed an excellent agreement. The anterior and posterior leaflets were constructed by identifying the leaflet free edge and points between the annulus and the free edge as described in Fig. 5a for several slices in the echocardiographic volume. The free edge and tissue points were mainly extracted from image planes rotating about the origin of the y-z plane (Fig. 5a), extracting points every 3-5 degrees. Moreover, as the commissures are difficult to extract, the volume was sliced from several different directions, to identify the commissural structure. All the identified points were then imported into the CAD software Rhino. In Rhino, the free edge was constructed by creating a non-uniform rational B-spline (NURBS) curve through the extracted free edge points, making a continuous line at the margin. Then, a NURBS surface was created by using the tissue points (see Fig. 5a) to guide the surface between the annulus and the free edge line. There was further identified three papillary muscle heads in the patient's echocardiography. One head was located anterolaterally (PM1A), and two heads were discovered to be situated in the posteromedial region. The papillary muscles in the posteromedial region 

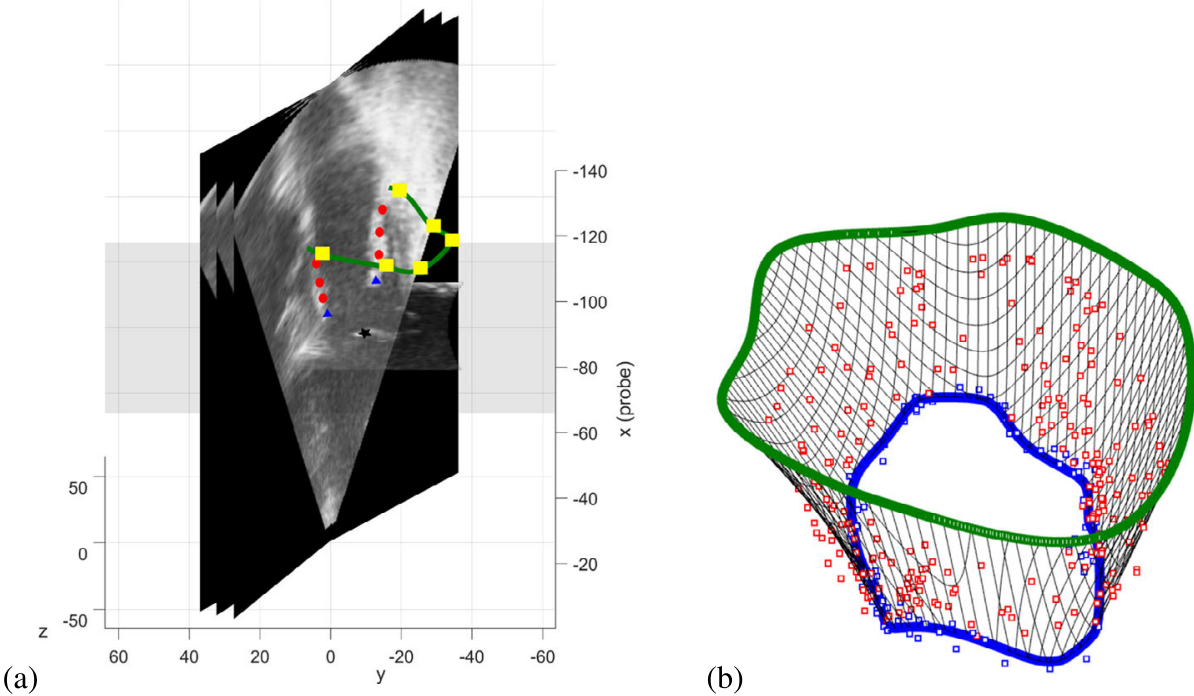

Fig. 5 (a) Explanation of the anatomical components on an echocardiographic image, at the end-diastolic configuration. Papillary muscle tip (star), leaflet edges (blue triangles), tissue points (red circles), annulus (green line), annulus interpolation points (yellow squares). (b) NURBS surface created from the extracted points, following the same coloring scheme as Fig. a. Anterior leaflet to the right, posterior leaflet to the left

were termed PM2A and PM2P. The PM2A has chordae inserting into the anterior leaflet, while the PM2P has chordae inserting into the posterior leaflet.

\subsection{Chordae Modeling}

Sixteen marginal chordae were modeled to originate from each papillary muscle. As the marginal chordae is thinner than the strut chordae, it is difficult to distinguish the marginal chordae on echocardiography. Hence, each marginal chordae is split in a fan-like manner, inserting into the leaflet free edge with about five different insertion points as described by [35]. The branching was mainly done in order to simulate a more anatomically correct model, where the branch origin was set between the papillary muscle and the free edge (Fig. 6a). Moreover, the branching led to a redistribution of stress on the leaflet edge, hence preventing excessive distorted elements in the analysis. Lastly, secondary (strut) chordae were discovered to insert into the belly of both the anterior and posterior leaflet, using a 3D view from the left ventricle in EchoPAC (GE Healthcare). As with the marginal chordae, the strut chordae branch out in a fan-like manner into the belly of both leaflets. The branching is distributed to the nearby nodes of the main insertion point in the FE model.

The circular cross-sectional areas for myxomatous marginal and strut chordae were modeled to be $1.6 \mathrm{~mm}^{2}$ and $2.22 \mathrm{~mm}^{2}$ respectively [7]. For the healthy human chordae, the cross-sectional areas were modeled to be $0.7 \mathrm{~mm}^{2}$ and $0.9 \mathrm{~mm}^{2}$ [7].

\subsection{Finite Element Model}

The end-diastolic patient-specific finite element geometry constructed from the echocardiographic data is depicted in Fig. 6. For the anterior and posterior leaflets, mainly reduced four noded general-purpose shell elements (S4R) were used. S3R elements were also used 


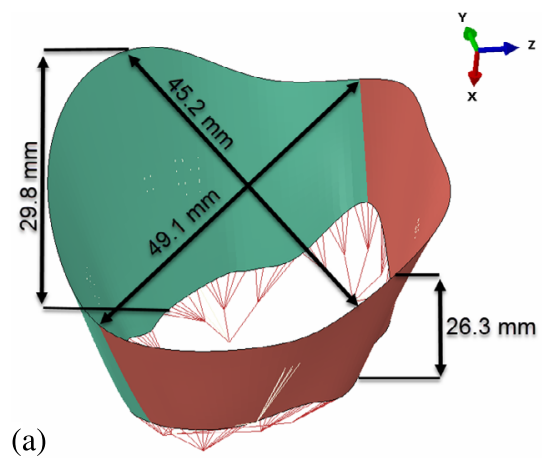

(b)

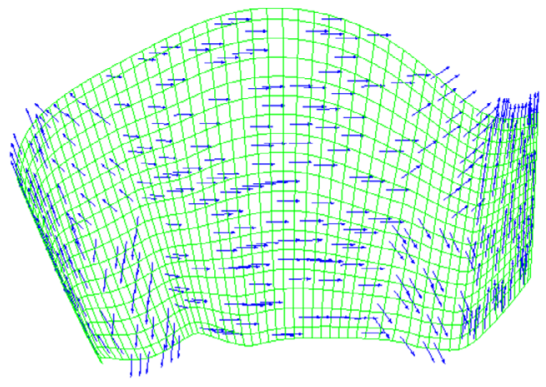

(c)
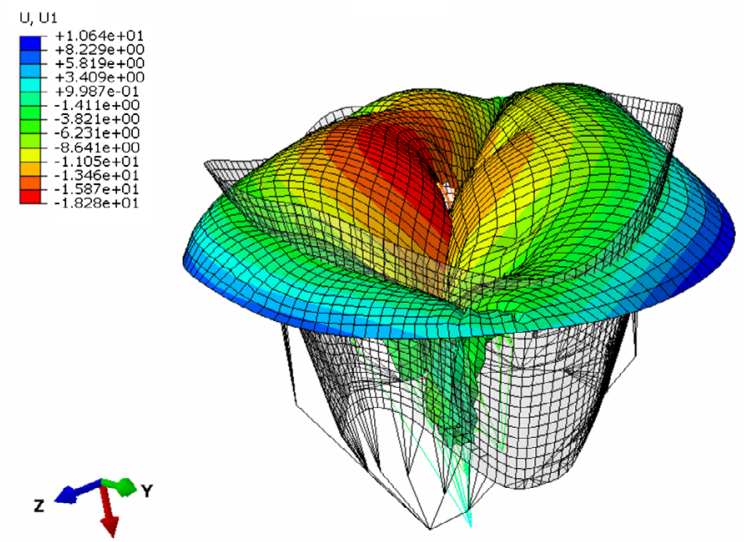

Fig. 6 (a) - Finite element model created from echocardiographic data. Anterior leaflet (green), posterior leaflet (red). Anterior leaflet height $29.8 \mathrm{~mm}$. Posterior leaflet height $26.3 \mathrm{~mm}$. Septal-lateral length 45.2 $\mathrm{mm}$. Commissure to commissure (CC) length $49.1 \mathrm{~mm}$. (b) - Anterior leaflet material orientation. (c) - EndDiastolic configuration (in gray) and late systolic configuration plotted with displacements $(\mathrm{mm})$ towards the atrium (x-direction)

in some geometrical regions of the leaflets. The chordae tendineae were modeled as twonoded 3D truss elements (T3D2). Furthermore, the overall leaflet thickness was set to be 3 $\mathrm{mm}$, which is the average thickness of Barlow leaflets found by [2]. As for contact, a general contact algorithm available in Abaqus was applied. The tangential contact behavior was set to frictionless and the normal contact behavior was a hard contact condition [25].

The material orientation applied to the mitral valve leaflets is obtained from small angle light scattering (SALS) data presented by [36]. In [36], the mean collagen fiber direction is observed to be perpendicular to the annulus near the commissures, and parallel to the annulus at the middle of the leaflets. Moreover, the fiber direction is observed to gradually rotate from parallel to perpendicular towards the commissures. Implementation of the fiber direction in Abaqus is done by partitioning the leaflet into several regions. Then a material orientation was assigned to each partitioned region (Fig. 6b).

\subsection{Boundary Conditions and Loading}

In order to simulate the dynamic valve closure from end-diastole to right before aortic valve closure (AVC), the Abaqus Explicit solver was used. The dynamics of the papillary muscle 
Fig. 7 Load amplitude curve from end-diastole to aortic valve closure (AVC). Amplitude Curve (AC), Aortic Valve Opening (AVO), Peak Pressure (PP), Mitral Regurgitation Start (MRS)

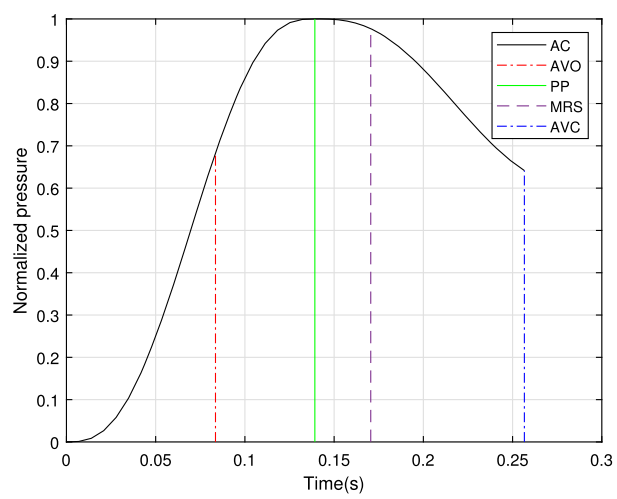

tips and the annulus were obtained using in vivo echocardiographic data. For all time frames, between end-diastole and AVC, the geometry of the annulus and the positions of the PMs are recorded. As it is difficult to track material points from echocardiographic measurements, the motion of the annulus was prescribed using displacement boundary conditions determined from the acquired images, under the assumption that heterogeneity in annular strain is small. First, the annular geometries of each time step were modeled as periodic degree-3 spline curves parameterized by arc length. Then, the relative parameterization of the annular curves was optimized to find the point-wise map that minimized the total displacement between two consecutive curves as described by Rego et al. [37]. Between each configuration a linear interpolation is performed, creating a continuous movement between the time frames. These boundary conditions are then implemented into the Abaqus user subroutine VDISP. In Fig. $6 c$ the end-diastolic configuration together with the deformed mitral valve configuration at AVC is presented, showing how the boundary conditions deform the mitral valve.

A uniformly distributed pressure was applied to the leaflets' ventricular surface, with a patient-specific peak pressure of $17.73 \mathrm{kPa}(133 \mathrm{mmHg})$. Furthermore, the amplitude was modeled in order to follow a pressure curve similar to the one during the cardiac cycle [38]. The pressure curve is presented in Fig. 7, where aortic valve opening (AVO), peak systole, mitral regurgitation start (MRS) and aortic valve closure (AVC) are marked.

\subsection{Material Property Cases}

In this study, there have been conducted several different FE analyses to study the effect of material behavior for both the leaflets and chordae. In Table 4 an overview of the FE analyses run, and what kind of combinations of material parameters for leaflet and chordae that are used are presented. The cases given the most focus are Cases 1, 2 and 3. In order to compare the material behavior on the Barlow FE geometry, only the material implementations are altered for the leaflets and not the thickness. From the study performed by [2], the mean leaflet thickness for Barlow patients were discovered to be $3 \mathrm{~mm}$. Hence this thickness was used for all analyses.

\subsection{Patient and Echocardiographic Measurement}

In this section the studied patient and her lesions are briefly described. As the patient is yet to have surgery, no information on the surgical procedure is presented. The studied subject gave 
Table 4 Material combinations applied to FE analyses

\begin{tabular}{lll}
\hline & Leaflet & Chordae \\
\hline Case 1 & Barlow [4] & Barlow [7] \\
Case 2 & Healthy [34] & Healthy [34] \\
Case 3 & Porcine [33] & Ovine [34] \\
Case 4 & Barlow [4] & Barlow [7] (No Strut) \\
Case 5 & Healthy [34] & Healthy [34] (No Strut) \\
Case 6 & Porcine [33] & Ovine [34] (No Strut) \\
Case 7 & Barlow [4] & Healthy [34] \\
Case 8 & Barlow [34] & Ovine [34] \\
Case 9 & Healthy [34] & Ovine [34] \\
Case 10 & Porcine [33] & Healthy [34] \\
Case 11 & Barlow [4] & Barlow [7] (constant \\
& & annulus after peak systole)
\end{tabular}

Fig. 8 Atrial view of the finite element mitral valve, with description of the leaflet segments. Anterolateral Commissure (AC), Posteromedial Commissure (PC), Anterior Horn (AH), Posterior Horn (PH)

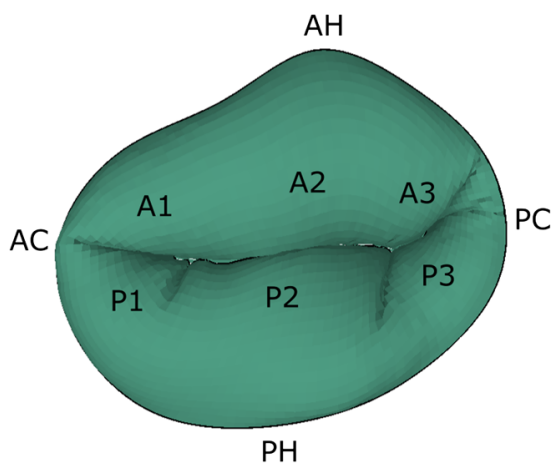

written informed consent, and the study protocol was approved by the Regional Committees on Biomedical Research Ethics.

The patient is a 53-year-old woman who was diagnosed with BD. The patient had a severely dilated annulus with excessive leaflet tissue and mitral regurgitation. From echocardiographic findings, multiple jets of mitral regurgitation in mid to late systole were observed. The most severe regurgitation was located in the central segments of the mitral valve (A2/P2 Fig. 8). There were also observed regurgitant jets near the P1 and P3 segments. The regurgitation at P1 was further observed to have the latest onset. Mitral annular disjunction (MAD) of $10 \mathrm{~mm}$ was observed in the posterior part of the annulus.

\section{Results}

\subsection{Comparison with Three-Dimensional Echocardiographic Data}

Validation of the FE model can be done by comparing the global response of the FE analysis with echocardiography. The FE mesh was imported into the echocardiographic recording at the onset of mitral regurgitation. At MRS, the FE analyses were compared with echocardiography from the posteromedial commissure (PC) to the anterolateral commissure (AC) (Fig. 9a) and from the anterior horn (AH) to the posterior horn (PH) (Fig. 9b). In Fig. 10, 

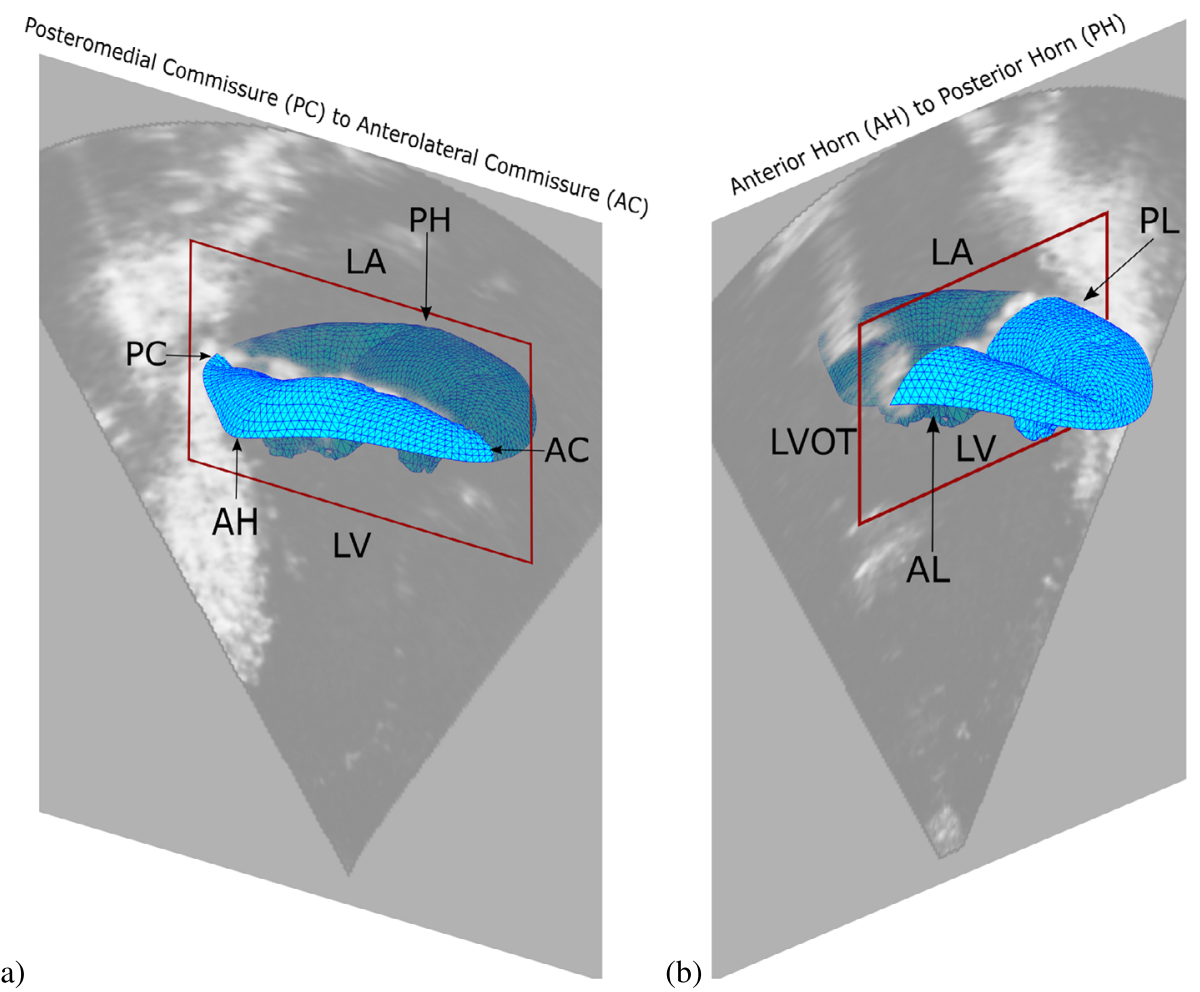

Fig. 9 FEA at MRS plotted on the echocardiographic slices. Red box describing the extracted frames in Fig. 10 and Fig. 11. (a)-Echocardiographic slice from the posteromedial commissure (PC) to the anterolateral commissure (AC). (b)-Echocardiographic slice from the anterior horn (AH) to posterior horn (PH). (LA)-Left Atrium, (LV)-Left Ventricle, (LVOT)-Left Ventricular Outflow Tract, (AL)-Anterior Leaflet, (PL)-Posterior Leaflet

the red boxes depicted in Figs. 9a-b are compared for the different material parameter cases presented in Table 4. Furthermore, each node from the FE analysis was projected onto the true (imaged) mitral valve geometry at MRS, enabling the calculation of the distance error between the FE analyses and echocardiography. At MRS the mid surface of the mitral valve was segmented from echocardiography together with a trained cardiologist. In Fig. 12, an atrial view of the FE meshes and each node's distance to echocardiography is presented for the material parameter cases.

As can be seen in Table 5, there is a good correspondence between the echocardiographic segmentation and the FE analyses with myxomatous and animal leaflet parameters, where the lowest mean point-to-mesh distance is observed for Case 1 with $1.67 \pm 1.21 \mathrm{~mm}$. For the AH-PH extraction the healthy material implementation (Case 2) has a flattened response, making the leaflet bulge less towards the left atrium compared to Cases 1 and 3 (Figs. 10a-b). Case 2 has the lowest displacement error in the anterior leaflet (Fig. 12), while the displacement error in the P2-P3 area is the largest due to the flattened response shown in Fig. 11b. For the posterior leaflet, Cases 1 and 3 manage to reproduce the bulging observed in the P2 segment (Figs. 10b and 12). However, both cases show large displacement errors in the P1 segment. Moreover, Cases 1 and 3 predict too much bulging of the anterior leaflet towards the left atrium compared to the echocardiographic measurements. When the strut chordae 
Table 5 Mean displacement errors and standard deviations from FE mesh to segmented atrial surface at MRS for Cases 1 to 8

\begin{tabular}{ll}
\hline & Mean \pm SD [mm] \\
\hline Case 1 & $1.67 \pm 1.21$ \\
Case 2 & $2.26 \pm 1.66$ \\
Case 3 & $1.92 \pm 1.38$ \\
Case 4 & $1.85 \pm 1.29$ \\
Case 5 & $2.20 \pm 1.63$ \\
Case 6 & $2.10 \pm 1.45$ \\
Case 7 & $1.81 \pm 1.32$ \\
Case 8 & $1.78 \pm 1.29$ \\
\hline
\end{tabular}

(a)

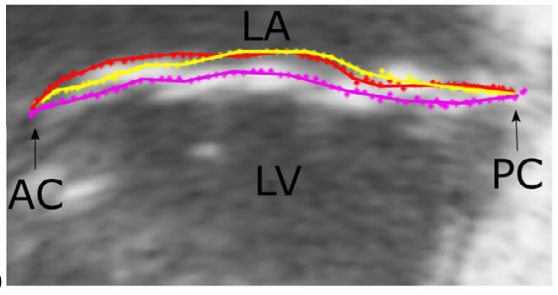

(b)

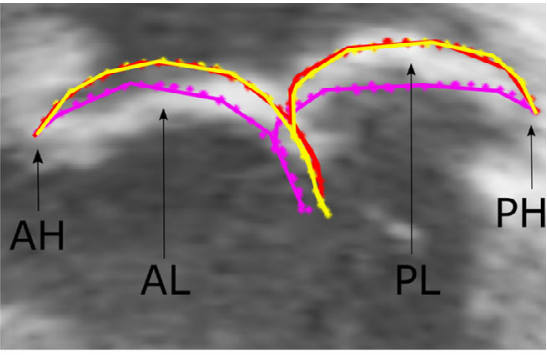

(c)

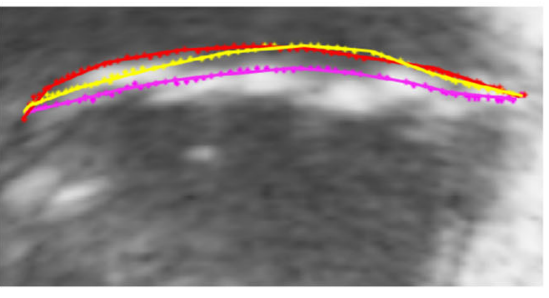

(e)

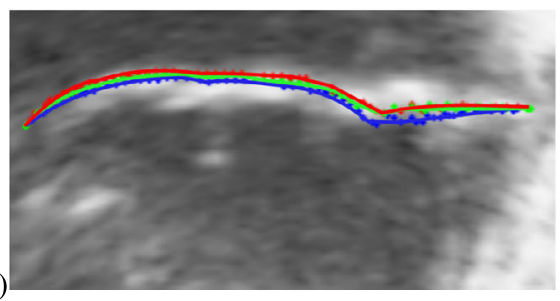

(d)

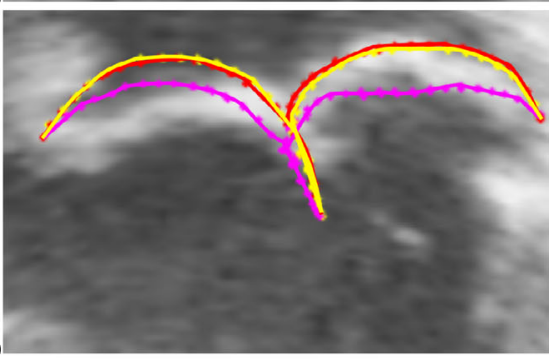

(f)

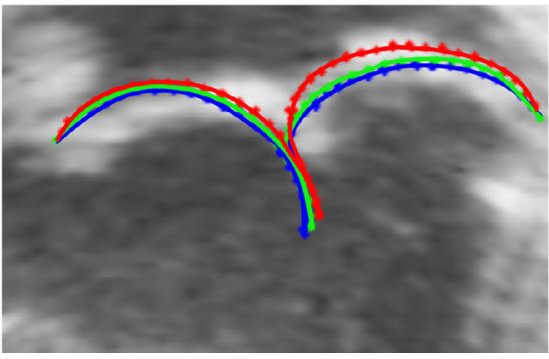

Fig. 10 Nodes along the leaflets from FE analysis plotted on echocardiographic 2D image at MRS. (a, c, e) shows a $2 \mathrm{D}$ extraction between the two commissures. $\mathrm{PC}$ to the right and $\mathrm{AC}$ to the left. (b, d, e) shows a view through the AH to the left and the PH to the right. (a, b)-Cases 1,2 and 3. (c, d)-Cases 4,5 and 6. (e, f)-Cases 1,7 and 8. Red - Myxomatous, Magenta - Healthy, Yellow - Porcine leaflet parameters for $(\mathbf{a}, \mathbf{b}, \mathbf{c}, \mathbf{d})$. Red Case 1, Blue - Case 7, Green - Case 8 for $(\mathbf{e}, \mathbf{f})$

are removed from the model (Cases 4-6), the bulging of the FE model increases, resulting in a poorer correspondence to echocardiography in all cases. Furthermore, in Figs. 10e-f the global response of the mitral valve model is similar. However, Case 7 has the smallest 
(a)

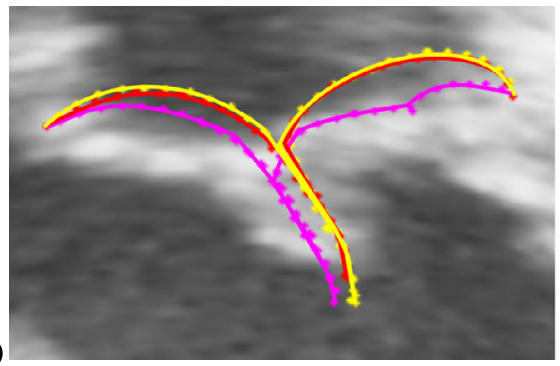

(b)

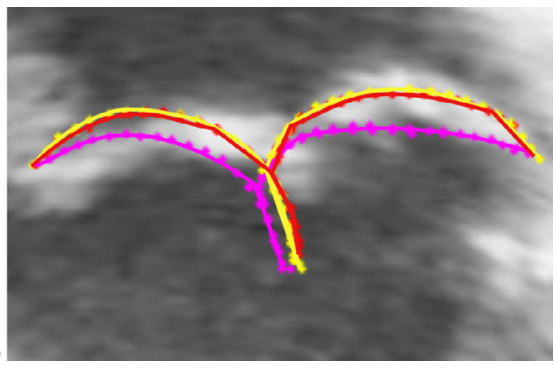

Fig. 11 Nodes along the leaflets from FE analysis plotted on echocardiographic 2D image, anterior to posterior horn view for Cases 1,2,3 at a-peak systole, b-late systole

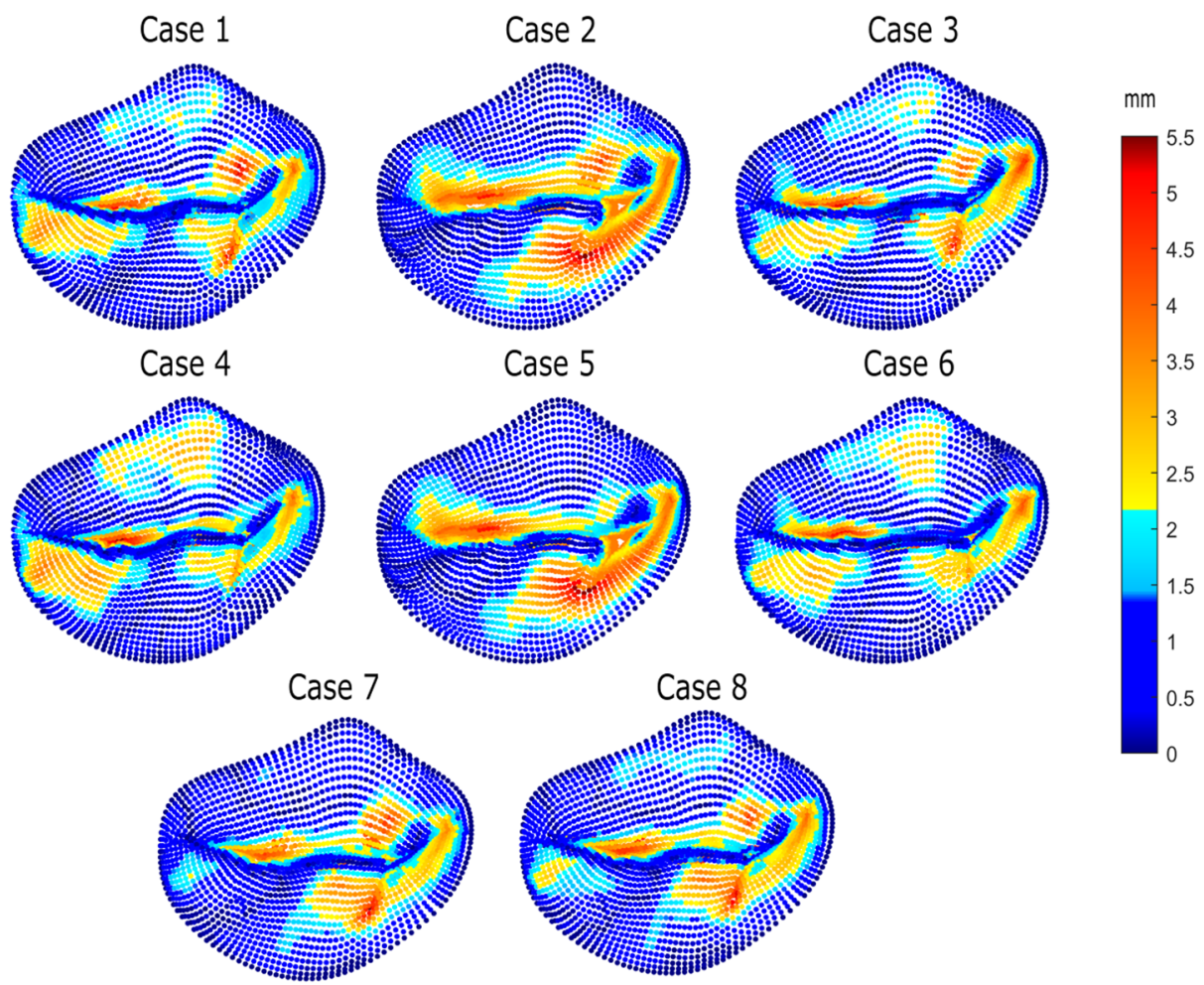

Fig. 12 Atrial view of the point-to-mesh distance errors (in $\mathrm{mm}$ ) between the FE mesh and the true (imaged) configuration at MRS

displacement error in the anterior leaflet and in the P1 segment compared to Case 1 and Case 8. Lastly, Fig. 11 compares Cases 1,2 and 3 with echocardiography at peak systole and late systole. This shows, that as ventricular systole progresses, the FE correspondence with echocardiography improves for Cases 1 and 3. The posterior leaflet in Case 2 has the best correspondence with echocardiography at peak systole. 

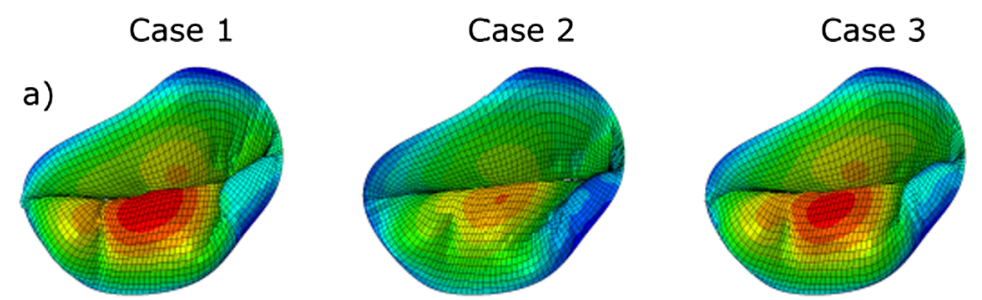

U, U1
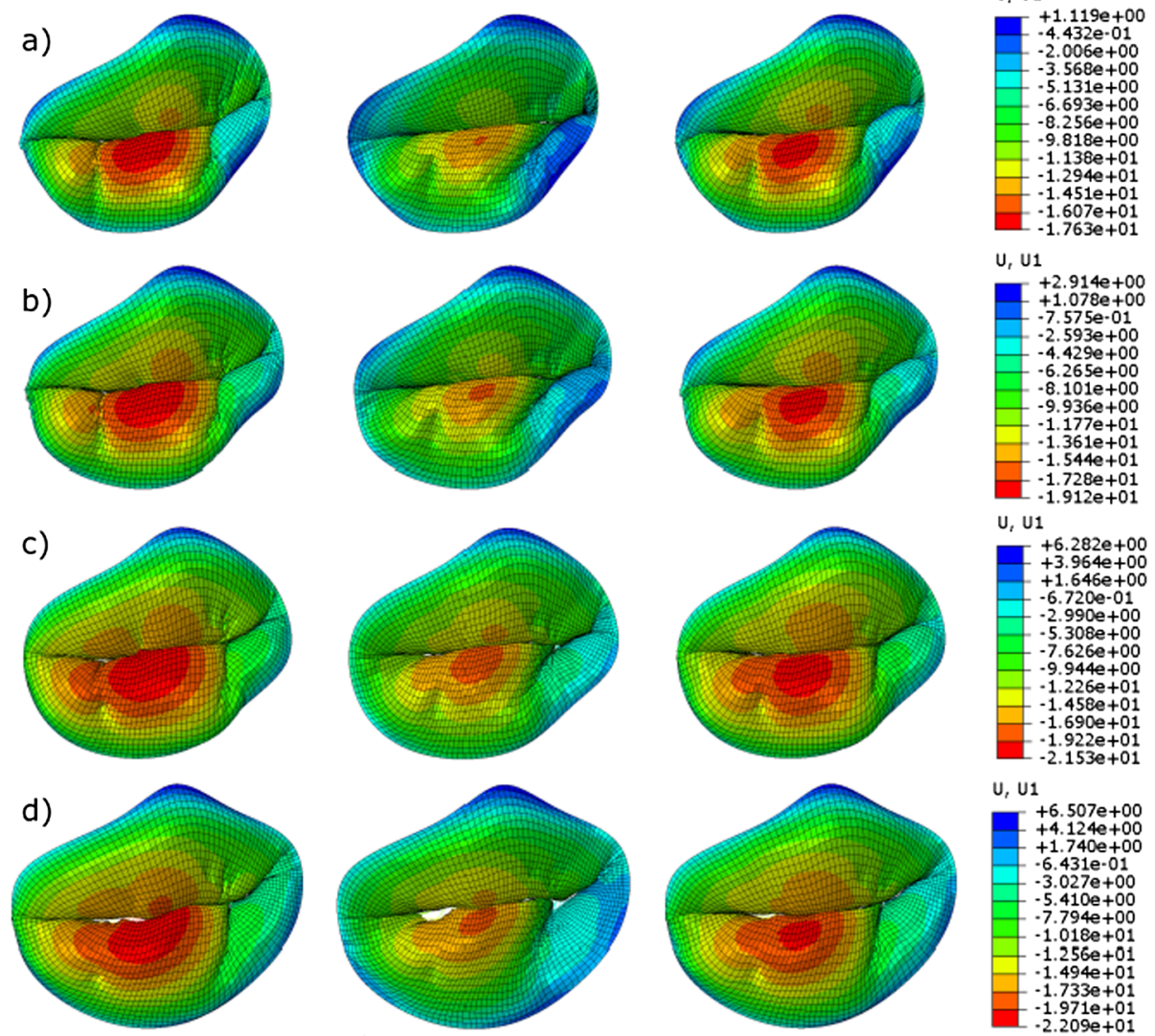

U, U1
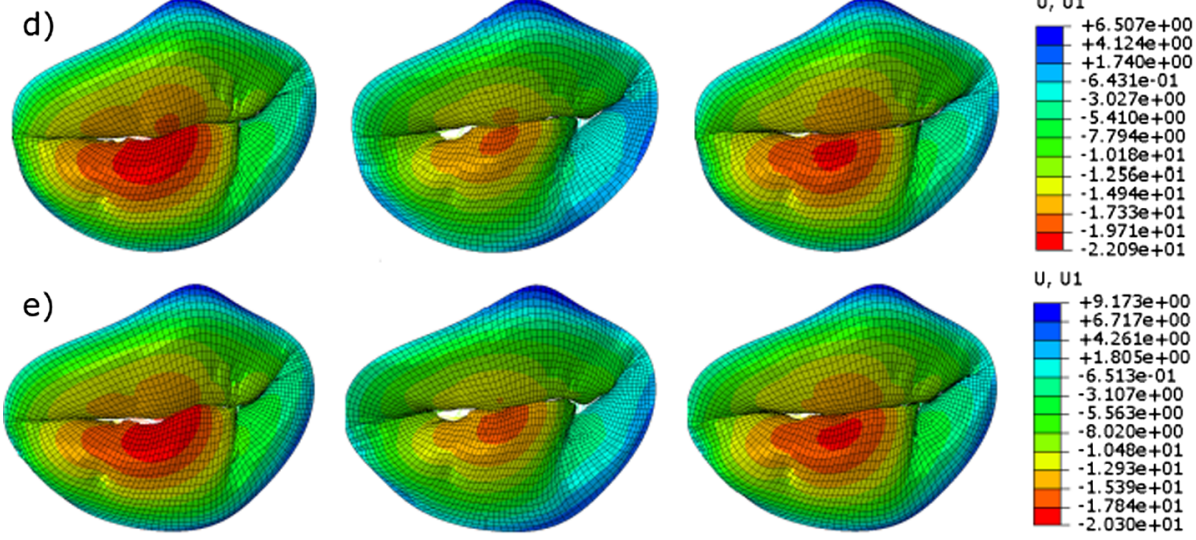

U, U1

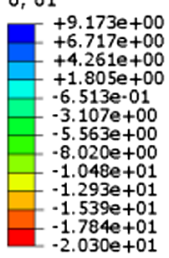

Fig. 13 Surgeons view of displacement in the U1-direction (x-axis Fig. 6) for different material parameter combinations. Displacements are extracted at: (a) $90 \mathrm{mmHg}$ (AVO $t=0.1044 \mathrm{~s}$ ). (b) $133 \mathrm{mmHg}$ (Peak Systole $t=0.1392 \mathrm{~s}$ ). (c) $129 \mathrm{mmHg}$ (MRS $t=0.174 \mathrm{~s}$ ). (d) $127 \mathrm{mmHg}$ (peak annular area $t=0.2088 \mathrm{~s}$ ). (e) $95 \mathrm{mmHg}(t=0.2436 \mathrm{~s})$. Case 1, Case 2 and Case 3 are described in Table 4

\subsection{Leaflet Displacement in Atrial Direction}

The atrial displacement (x-direction in Fig. 6) for Cases 1, 2 and 3 (Table 4) are presented in Fig. 13. The variation in material properties clearly leads to varying atrial displacements, where the healthy material implementation (Case 2) displaces the least towards the left atrium. Cases 1 and 3 have a similar atrial displacement for the anterior leaflet, while Case 1 shows most displacement of the posterior leaflet especially near the free edge of the P2 segment. It is further showed that at the onset of mitral regurgitation (Fig. 13c), tendencies 

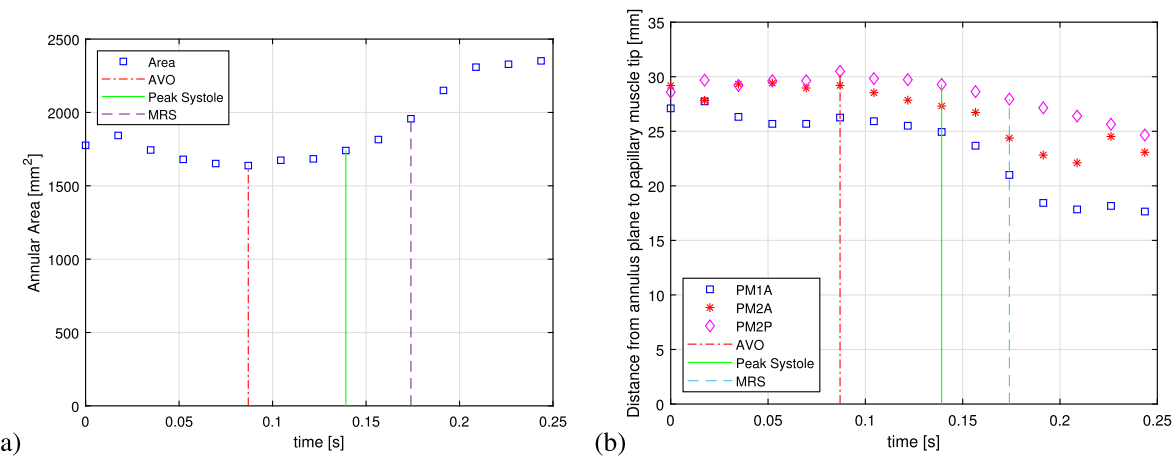

Fig. 14 (a)-Annular area for each time point from end-diastole to end of analysis $(t=0.2523 \mathrm{~s})$. (b)-Distance from annular plane to papillary muscle tips at each time point from end-diastole to end of analysis. PM1A being the one PM head at the anterolateral side. PM2A and PM2P are the two observed heads on the posteromedial side. All results are extracted from echocardiography, capturing the dynamics of the annulus and papillary muscles

to a leakage near the anterolateral commissure is observed for Cases 1 and 2. At peak annular dilation (Fig. 13d), Cases 1 and 2 have leakage at the anterolateral commissure, while Case 2 also shows leakage at the posteromedial commissure. During the late systolic phase (Fig. 13e), the leakage in both commissures for Case 2 has increased in severity. Moreover, there is observed a prolapse of the P2 segment for Cases 1 and 2, where the segment overrides the anterior leaflet.

\subsection{Annular Area - Distance from PM to Annulus}

For each time step, a mean annulus plane was calculated from the annular spline extracted from echocardiography. Then, the annulus was projected onto this mean plane and a twodimensional (2D) area was computed and plotted against time in Fig. 14a. This plot shows how much the annulus dilates during the analysis, with an increase in annular area of $43 \%$ from AVO to the last time-frame. At peak systole it is observed that the annular area starts to increase and continues to do so until the end of the analysis. Furthermore, after peak systole the papillary muscle tips move towards the mean annulus plane (Fig. 14b), where the anterolateral papillary muscle (PM1A) has the most substantial displacement towards the atrium after peak systole.

\subsection{Total Area in Contact}

To compare the different FE analyses, it is of interest to extract the total area in contact (CAREA) from the FE model. The contact area is defined by the area of elements that are in contact during the FE analysis time. It can be interpreted as the coaptation zone of the leaflets. For the cases presented in Table 4, except the ones with no strut chordae, the computed contact area is extracted and plotted in Fig. 15.

At the onset of mitral regurgitation, the CAREA for all the analyses presented are shown to decrease rapidly. It is evident that the rate of CAREA decrease is independent of material implementation. However, the amount of leaflet area coapting can be shown to be dependent on both leaflet and chordae material combination. The dependency on chordae material property implementation can be observed in Figs. 15a-c, where healthy human chordae material parameters have been analyzed with different leaflet material parameters (Case 2, Case 


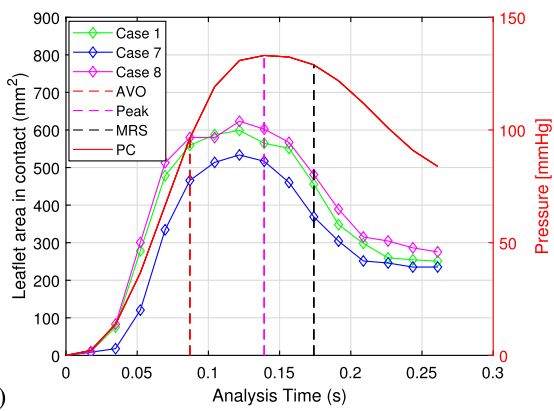

(a)

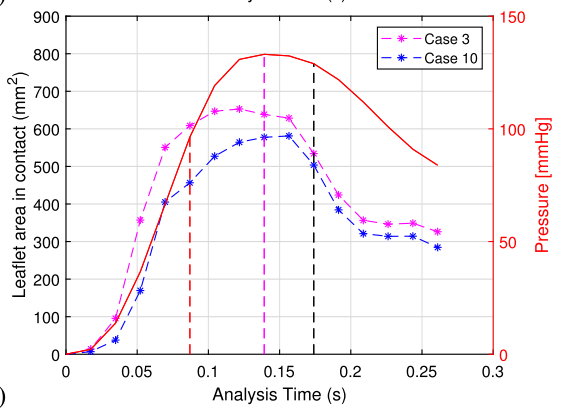

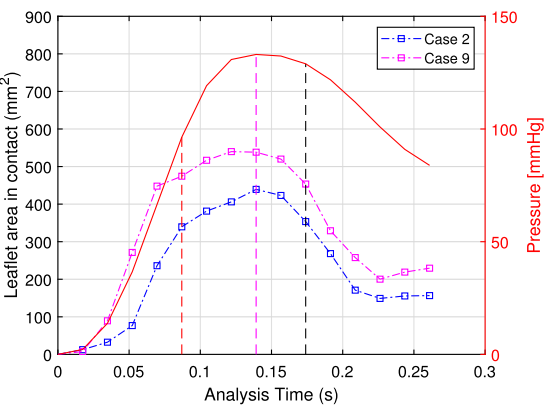

(b)

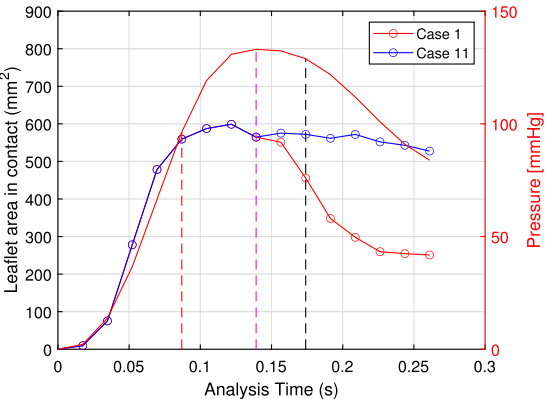

Fig. 15 Contact area (CAREA) of the mitral valve leaflets with different material combinations (see Table 4) for the leaflets and chordae plotted together with the pressure curve (PC)

Fig. 16 Contact area (CAREA) of Case 1 plotted together with the annular area from end-diastole to end of analysis $(t=0.2523 \mathrm{~s})$

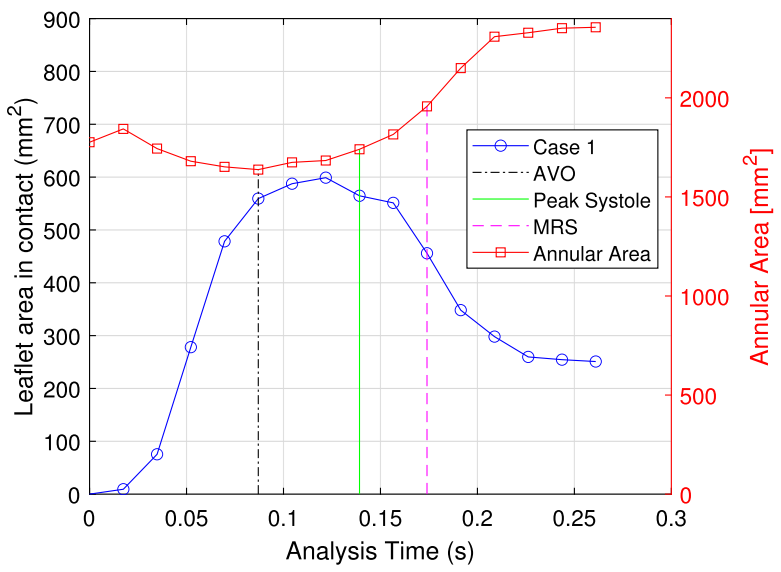

7 and Case 10). When healthy human chordae material properties are implemented together with myxomatous leaflet parameters (Case 7), CAREA is 10\% less compared to the Case when myxomatous properties are used for both leaflets and chordae (Case 1) at peak systole (Fig. 15a). Studying the effect of leaflet material implementation only, Case 10 has $31 \%$ more contact area compared to Case 2 at peak systole. In Fig. 15d, the effect of the annular dilation is investigated by keeping the annulus geometry constant after peak systole (Case 11). With the annular geometry constant, the abrupt CAREA decrease after MRS is eliminated and the CAREA is maintained until the end of analysis. Lastly, in Fig. 16 the CAREA 


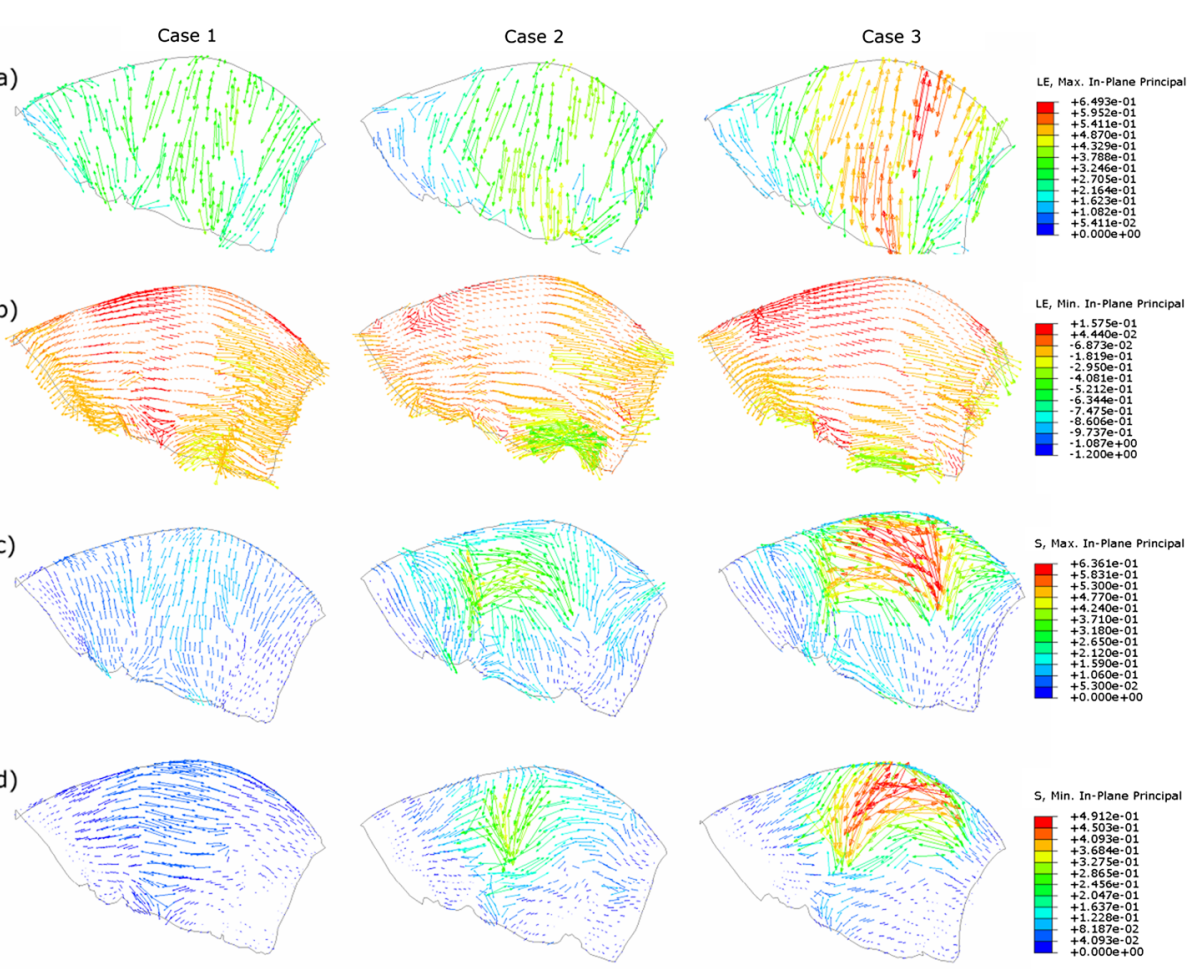

Fig. 17 The maximum and minimum, in-plane principal stresses (MPa) and logarithmic strains (LE) for the anterior leaflet at peak systolic pressure

is plotted together with the annular area, showing how the annular area increase correlates with the CAREA decrease.

\subsection{Stresses and Strain}

The maximum and minimum, in-plane principal stresses and strains for Cases 1,2 and 3 are plotted on the anterior leaflet at peak systole in Fig. 17. The maximum strain is observed to be perpendicular to the assigned fiber direction (radial direction) in the central region of the leaflet for all studied cases (Fig. 17a). Furthermore, Case 3 is shown to have the highest values of logarithmic strain, especially in the right portion of the A2 segment. In the circumferential direction, the anterior leaflet shows compressive behavior where the leaflets fold. The folding of the leaflets is mainly observed near the commissural regions, but folding is also discovered near the strut and marginal chordae insertion sites. Near the free edge between the A2 and A3 segment, compressive strains are observed due to folding caused by the marginal chordae for all cases. The circumferential strains in this region is the lowest for Case 2 (Fig. 17b). For the maximum and minimum in-plane principal stress, Case 3 has the highest values in the A2 segment close to the annulus (Figs. 17c-d.).

\subsection{Parametric Study of the $\kappa$ Parameter}

A parametric study on the dispersion parameter $\kappa$ was conducted to study how a change in $\kappa$ would affect the FE model to echocardiography correspondence. For the fitted Barlow 
Table 6 Mean displacement errors and standard deviations from FE mesh to segmented atrial surface at MRS for varying $\kappa$

\begin{tabular}{ll}
\hline & Mean \pm SD [mm] \\
\hline$\kappa=0.175$ & $1.93 \pm 1.41$ \\
$\kappa=0.2$ & $1.87 \pm 1.37$ \\
$\kappa=0.225$ & $1.82 \pm 1.37$ \\
$\kappa=0.25$ & $1.86 \pm 1.37$ \\
$\kappa=0.275$ & $1.73 \pm 1.26$ \\
$\kappa=0.3$ & $1.70 \pm 1.24$ \\
$\kappa=0.316$ & $1.67 \pm 1.21$ \\
$\kappa=0.33$ & $1.75 \pm 1.26$ \\
\hline
\end{tabular}

material parameters (Case 1), the $\kappa$ parameter was changed from 0.175 to 0.33 increasing towards an isotropic dispersion. The mean displacement error between each FE analysis and the true (imaged) mitral valve at MRS was calculated and presented in Table 6. The displacement error is slightly decreasing with increasing $\kappa$.

\section{Discussion}

\subsection{Influence of Material Behavior}

The material parameters acquired from the fitting procedures described in Sect. 2.4 have been implemented in the FE model and combined in various cases as described in Table 4. From Figs. 3 a and c, the myxomatous tissue was shown to be more extensible compared to the healthy human and porcine tissue when subjected to an equibiaxial strain state. These results were consistent with findings in literature, where myxomatous leaflet tissue was identified to be twice as extensible compared to healthy human leaflets [4]. From the off-biaxial test in Fig. 3 the porcine and Barlow leaflet parameters were shown to have the most extensible response in the radial direction. However, the logarithmic strains in the radial direction for Case 3 at peak systole were unphysiological, with strains reaching 0.91 in the radial direction. In previous papers $[16,39]$, the radial strains in the belly region of the anterior leaflet were measured to range from 0.25-0.3. Towards the trigones, Stevanella et al. [39] reported radial strains of up to 0.45 . In all our modeled cases, the measured radial strain was above the ranges reported in the belly region. Nevertheless, due to the excessive dilation of the annular area, size of the leaflets and abnormal dynamics, the large strain values in the radial direction for Case 1 and Case 2 were not surprising. The unphysiological strains observed for Case 3 however, implies that the material parameters used for the porcine leaflet might be unsuitable for modeling Barlow mitral valves. In the circumferential direction, compressive strains were observed in areas where the leaflets folded. The compressive strains in the commissures ranged from $(-0.06$ to -0.16$)$, which is consistent with the findings in [39]. However, near the free edge in the A2-A3, the compressive strains were observed to reach -0.4 for Case 2 at peak systole. At this location, there is a complex load scenario at peak systole, with high pressures, tight chordae and coapting leaflets which facilitates this behavior.

Previous studies have shown that the anterior leaflet is stiffer compared to the posterior leaflet $[40,41]$. From the comparison of the FE analyses with echocardiography (see Fig. 12), Case 2 shows the best correlation with the anterior belly region excluding the coaptation zone. It can be observed that the stiffer healthy human material parameters contribute 
to less bulging of the anterior leaflet. As little is known regarding the mechanical properties of a myxomatous anterior leaflet, there is no distinction between the material properties applied to the leaflets in the FE model used in Case 1. Applying healthy human material parameters for the anterior leaflet in Case 1, would decrease the point-to-mesh distance error in the anterior leaflet. This indicates that the myxomatous anterior leaflet may be stiffer than the myxomatous posterior leaflet. Case 7 shows that also healthy human chordae material parameter contributes to a locally smaller point-to-mesh distance error for both the A2 and P1 region. Nevertheless, the mean point-to-mesh distance error is still lower for Case 1 , where all Barlow parameters are implemented. It is further shown that as we move from peak systole to late systole, the FE correspondence with echocardiography improves (see Fig. 11). The annular dilation after MRS stretches the leaflets in place, and the deformations computed by the FE analysis improves its alignment to the patient's in vivo response. However, at peak systole (Fig. 11a) the FE model is not able to reproduce the echocardiographic response of the anterior leaflet for Cases 1 and 3, predicting more bulging towards the left atrium.

The maximum and minimum principal stresses observed in the leaflet with the myxomatous material implementation are shown in Fig. 17 to follow the direction of the maximum and minimum logarithmic strain. This behavior is caused by the dispersion parameter $\kappa$ being close to $1 / 3$ in the GOH-material model, which corresponds to an isotropic distribution. Having a $\kappa$ parameter near $1 / 3$ for myxoid tissue is further a good assumption, as the collagen orientation is observed to be disrupted [8-11], giving the tissue a more isotropic behavior. From the parametric study conducted in Sect. 3.6, the mean displacement error decreased when $\kappa$ approached 1/3. Showing that the diseased mitral valve presented in this study fits better with the FE model when the material model is nearly isotropic. Moreover, the myxomatous leaflet tissue is discovered to be almost equally extensible in the circumferential and radial direction [4], which further exemplifies that the myxomatous leaflets have a quasi-isotropic material response. For the myxomatous leaflet tissue, the material parameters are acquired by an inverse FE procedure based on uniaxial tests performed in [4]. The extension tests provided by Barber et al. [4] in the radial and circumferential directions are used simultaneously in the fitting procedure as recommended by Holzapfel [42] in order to describe the material response in the physiological domain. Biaxial tests on myxomatous tissue would be favorable, however to our knowledge no such study has yet been conducted.

During ventricular systole the anterior leaflet is shown to stretch far more in the radial direction compared to the circumferential direction [43]. It is shown that having a more extensible material behavior (Cases 1 and 3) leads to larger CAREA values (see Fig. 15). The stiff healthy tissue implementation (Case 2) results in the smallest amount of coaptation area and atrial displacements, which again leads to the most severe malcoaptation. For the myxomatous implementation, the coaptation area is increased compared to the healthy tissue, due to the myxomatous tissue being softer. In general, it seems like the remodeling of the mitral valve for patients with $\mathrm{BD}$, especially the reduction of leaflet stiffness, may help to protect the valve from a bigger leak.

\subsection{Annular Area}

Severe annular dilation during systole is a characteristic feature for patients with BD [44]. For the studied patient in this paper, the increase in annular area from AVO to peak annular area was reported to be $43 \%$. Furthermore, the onset of mitral regurgitation was observed during the time interval where the annular area increased the most (Fig. 14b). During this time interval, the contact area for all FE analyses had an average decrease of $49 \%$ from MRS 
to end of analysis, emphasizing how the annular dilation affects the mitral valve leaflets ability to coapt. For the analysis where the annular dilation was stopped after peak systole (Case 11), the abrupt decrease in contact area was eliminated (Fig. 14d). This clearly shows why an annuloplasty ring is important during Barlow mitral valve repair in order to prevent regurgitation due to excessive annular dilation. Even though the area in contact was observed to remain constant as the pressure decreased in Fig. 14d, it is important to note that the annular and papillary muscle dynamics were modeled after the patient's echocardiographic recordings, i.e., for a given pressure history. Therefore, we can only conclude that stopping the annular dilation helps prevent malcoaptation, but not that it is pressure independent. The leaflets themselves are closing at very low pressures. Hence, it is not surprising that there is almost no alteration in coaptation area due to the pressure decrease after peak systole.

\subsection{Chordae Modeling}

The marginal chordae tendineae were modeled so that they originate from the papillary muscles and insert into the free edge of the mitral valve leaflets, while the strut chordae insert into the leaflet rough zone. The importance of chordae modeling has been investigated earlier by Wang and Sun [16] and was also in this paper shown to be important for atrial bulging of the mitral valve leaflets (see Cases 4-6 Fig. 12). From echocardiographic images, it was not possible to get a full representation of the number of chordae inserting into the free edge nor its insertion site. Thus, the modeling of marginal chordae was assumed from literature [35]. For the strut chordae however, it was possible to estimate the location of the strut chordae insertion sites for both leaflets by studying a ventricular view of a 3D rendering in EchoPAC (GE Healthcare) at peak systole. For Cases 1,7 and 8 only the chordae parameters were changed for each analysis. In terms of global response (Fig. 13) and mean displacement errors (Table 5), there was discovered a negligible difference for these analyses, showing that the mechanical properties of the mitral valve chordae were not so important as long as they are kept within the physiological limits.

\subsection{Constitutive Material Modeling}

A model such as the one developed in [47], including a continuous fiber distribution and some recruitment function of the collagen fibers, may be a better approach for modeling mitral valve mechanics. In this study, we choose to use a simpler approach by using the GOH-model which has a discrete fiber distribution. The issue was raised in the study by [46], where it was shown that the structural simplifications of such a model are unable to predict mechanical data as accurately as the models accounting for a continuous orientation distribution function. However, Bischoff [46] also reported that "The mechanical behavior of tissues that demonstrate unimodal distributions (e.g., pericardium, mitral valve, and tendon/ligament) can thus be accurately modeled using a discrete representation in place of its continuous analog". Furthermore, [37] discovered that modeling the mitral valve with the Ogden model (12) resulted in less than $3 \%$ error in the estimation of circumferential and radial stretches. This small error was in the study assumed to be due to the lack of axialcoupling. Hence, our simpler approach for the constitutive material model of the mitral valve leaflets may be sufficient. As little is known regarding the microstructure of myxomatous mitral valve leaflets (i.e., mean fiber direction and collagen dispersion), we did not want to over-engineer our modeling approach of the mitral valve by introducing more unknown parameters and functions. In this work, the mean fiber orientation was assumed - following the work done by [36] and the dispersion parameter $\kappa$ was calibrated from the available 
experimental data. When more information concerning the microstructure of myxomatous tissue, unimodal continuous models should be considered. Due to the equal extensibility of the radial and circumferential direction and the evidence of disorganized collagen, seen in both humans $[8,9]$ and dogs $[10,11]$, we can assume that the myxomatous tissue is rather isotropic. Therefore, an anisotropic material model may not be necessary to model the mechanical behavior of the diseased tissue. However, healthy human and porcine mitral valve leaflets were reported to exhibit an anisotropic mechanical behavior [33, 45]. For the comparison of the response of our FE model using myxomatous, human or porcine leaflets, we used the same material model $(\mathrm{GOH})$ for every analysis and thus only the material parameters are varying.

Additionally, Laurence et al. [48] investigated the regional differences of the mechanical properties of porcine mitral and tricuspid valves. For the mitral valve leaflets, they discovered regional differences, where all regions showed an anisotropic behavior especially in the belly region. In the study by Hadian et al. [11], the microstructure of myxomatous canine mitral valves was investigated. The study showed that there were regional differences in fiber distribution: the regions with myxomatous infiltration had disorganized collagen fibrils with no distinct orientation, while non-myxomatous regions had a distinct preferred collagen orientation. Both studies $[11,48]$ illustrate the limitation of the common assumption of modeling the leaflets with homogeneous material properties. However, there are limited possibilities to study the mechanical properties of all myxomatous leaflet segments for human specimens [7], thus homogeneous material properties are also assumed in this work for the mitral valve leaflets. To create a material model that incorporates the regional difference of material properties and microstructure, we need an in vitro study such as the one done in [27]. To overcome this limitation, we have to make simplifications and assumptions that still can manage to replicate the mitral valve in vivo response.

\subsection{Chordae Pre-Tension and Leaflet Pre-Strain}

For the mitral valve model presented in this study, it was not possible to detect if the chordae were stretched or not in diastole, hence it was difficult to assume if the chordae had some form of pre-tension. A characteristic feature for Barlow patients is the elongated chordae at systole [44], which might leave the chordae slack during diastole. It was therefore assumed that there was no pre-tension in the chordae for the patient-specific FE model of the studied Barlow patient. Furthermore, based on the small difference in mean displacement error between Cases 1,7 and 8 and if we think of the healthy human chordae parameters (2) as pre-tensioned chordae compared to the other material implementations, the Barlow mitral valve global response may not be very much influenced by chordal pre-tension. Nevertheless, as shown in Fig. 12 the human chordae parameters seem to slightly improve the correspondence to echo locally (at segments A2 and P1) compared to Case 1. This suggests that calibrating the chordae with pre-tension forces or material parameters to fit the fully coapted imaged configuration could potentially improve the model as done in previous studies [45, 49]. In the study by Pham et al. [45] a patient-specific mitral valve diagnosed with functional mitral regurgitation (FMR) was modeled in a finite element framework. Without adjustments of both chordae length and chordae pre-tension, their model did not manage to reproduce the global in vivo response of the patient. Therefore, they tried to minimize the point-to-mesh distance error by applying chordal tethering forces and optimizing the chordae length. However, FMR is not a degenerative disease and in [45] hypokinesis and leaflet tethering was observed in the patient. Hence, evidence of increased tension in the subvalvular apparatus were present. In the study by Drach et al. [49], the chordae were pretensioned by calibrating each chordae material parameter to reproduce the morphed fully 
coapted leaflet configuration. This procedure was made possible using fiducial markers and micro-CT which cannot be used in a clinical setting. Furthermore, as this study was an in vitro study, they did not account for the papillary muscle movement and annular dynamics which also plays a crucial role in the coaptation of the mitral valve leaflets. As shown in this study, the leaflet material properties have a somewhat large influence on the valve response. Therefore, calibrating chordae pre-tension and material parameters could be done when the material behavior of the leaflets is well known.

Moreover, in this work, we used material constitutive laws fitted to ex vivo experimental tests. However, there is evidence of components capable of active contraction such as muscle fibers, muscle cells, and innervation inside the leaflets' microstructure [50-61]. In addition, Amini et al. [62] reported a significant shrinkage of the mitral anterior leaflet in sheep when it is excised from the heart. Rausch et al. [63] used a nonlinear inverse finite element analysis to show that the stiffness of the anterior leaflet is highly sensitive to the level of pre-strain employed and that the discrepancy between in vitro and in vivo measurements of mitral valve stiffness can be explained by the presence of pre-strains. In addition, our previous studies $[50,64]$ suggested that the active components present in the mitral leaflets and the presence of pre-strains contribute to the physiological deformations and position of the mitral valve at peak systole. However, pre-strain and active muscle force levels are still unknown in healthy and diseased human mitral valves.

\subsection{Final Remarks}

Comparing the patients lesions with the FE analyses performed in this paper, we observe some similarities. The FE model in Case 1 observed lack of closure due to a prolapsed segment in the $\mathrm{P} 2$ region, coinciding with the most severe regurgitation observed from echocardiographic Doppler of the patient. Moreover, our patient experienced a late systolic regurgitation in the anterolateral commissure, which may coincide with the increased lack of closure in the FE model in late systole (see Fig. 13). In Fig. 14b, the anterolateral papillary muscle was observed to displace towards the mean annular plane in systole, which might affect the leakage in the anterolateral commissure. In the P3 segment, we also observed tendencies to leakage for Case 1, however not as severe as from Case 2. Nevertheless, several patients have to be studied in order to conclude that our model is able to predict the regurgitation observed in vivo.

In the present study, we managed to estimate the location of the chordae insertion into the middle of the posterior and anterior leaflet. However, as discussed earlier, the number of marginal chordae and insertion sites were assumed. In the validated mitral valve model developed by Wang and Sun [16], the representation of the chordae was acquired by multislice computed tomography (MSCT). This imaging modality enabled them to represent the chordal origins and insertion points, making their model able to replicate the in vivo behavior excellently. Due to the abnormal systolic changes in the mitral valve apparatus observed in Barlow patient's, we needed to use echocardiography for the segmentation of the mitral valve. In the future, imaging modalities such as CT could be combined with echocardiography to refine our model of the mitral apparatus.

Acknowledgements We thank Dr. Gabriel Kiss from the Norwegian University of Science and Technology for converting the echocardiographic recording in a suitable HDF5 format. This project was funded by the Trond Mohn Foundation (TMS2019TMT09).

Funding Note Open access funding provided by NTNU Norwegian University of Science and Technology (incl St. Olavs Hospital - Trondheim University Hospital). 


\section{Declarations}

Conflict of Interest The authors declare that they have no conflict of interest.

Open Access This article is licensed under a Creative Commons Attribution 4.0 International License, which permits use, sharing, adaptation, distribution and reproduction in any medium or format, as long as you give appropriate credit to the original author(s) and the source, provide a link to the Creative Commons licence, and indicate if changes were made. The images or other third party material in this article are included in the article's Creative Commons licence, unless indicated otherwise in a credit line to the material. If material is not included in the article's Creative Commons licence and your intended use is not permitted by statutory regulation or exceeds the permitted use, you will need to obtain permission directly from the copyright holder. To view a copy of this licence, visit http://creativecommons.org/licenses/by/4.0/.

\section{References}

1. Iung, B., Baron, G., Tornos, P., Gohlke-Barwolf, C., Butchart, E.G., Vahanian, A.: Valvular heart disease in the community: a European experience. Curr. Probl. Cardiol. 32(11), 609-661 (2007)

2. Hjortnaes, J., Keegan, J., Bruneval, P., Schwartz, E., Schoen, F.J., Carpentier, A., Levine, R.A., Hagege, A., Aikawa, E.: Comparative histopathological analysis of mitral valves in Barlow disease and fibroelastic deficiency. Semin. Thorac. Cardiovasc. Surg. 28(4), 757-767 (2016)

3. Anyanwu, A.C., Adams, D.H.: Etiologic classification of degenerative mitral valve disease: Barlow's disease and fibroelastic deficiency. Semin. Thorac. Cardiovasc. Surg. 19(2), 90-96 (2007)

4. Barber, J.E., Kasper, F.K., Ratliff, N.B., Cosgrove, D.M., Griffin, B.P., Vesely, I.: Mechanical properties of myxomatous mitral valves. J. Thorac. Cardiovasc. Surg. 122(5), 955-962 (2001)

5. Apor, A., Nagy, A.I., Kovacs, A., Manouras, A., Andrassy, P., Merkely, B.: Three-dimensional dynamic morphology of the mitral valve in different forms of mitral valve prolapse - potential implications for annuloplasty ring selection. Cardiovasc. Ultrasound 14(1), 32 (2016)

6. Fornes, P., Heudes, D., Fuzellier, J., Tixier, D., Bruneval, P., Carpentier, A.: Correlation between clinical and histologic patterns of degenerative mitral valve insufficiency: a histomorphometric study of 130 excised segments. Cardiovasc. Pathol. 8(2), 81-92 (1999)

7. Barber, J.E., Ratliff, N.B., Cosgrove, D.M., Griffin, B.P., Vesely, I.: Myxomatous mitral valve chordae. I: mechanical properties. J. Heart Valve Dis. 122, 955-962 (2001)

8. Kern, W.H., Tucker, B.L.: Myxoid changes in cardiac valves: pathologic, clinical, and ultrastructural studies. Am. Heart J. 84(3), 294-301 (1972)

9. Pellerin, D., Brecker, S., Veyrat, C.: Degenerative mitral valve disease with emphasis on mitral valve prolapse. Heart 84(4), 20-28 (2002)

10. Fox, P.R.: Pathology of myxomatous mitral valve disease in the dog. J. Vet. Cardiol. 14(1), 103-126 (2012)

11. Hadian, M., Corcoran, B.M., Han, R.I., Grossman, G.J., Bradshaw, J.P.: Collagen organization in canine myxomatous mitral valve disease: an X-ray diffraction study. Biophys. J. 93(7), 2472-2476 (2007)

12. Cole, W.G., Chan, D., Hickeyt, A., Wilckent, D.E.L.: Collagen composition of normal and myxomatous human mitral heart valves. Biochem. J. 219(2), 451-460 (1984)

13. Kunzelman, K.S., Cochran, R.P., Chuong, C., Ring, W.S., Verrier, E.D., Eberhart, R.D.: Finite element analysis of the mitral valve. J. Heart Valve Dis. 2(3), 326-340 (1993)

14. Prot, V., Skallerud, B., Holzapfel, G.A.: Transversely isotropic membrane shells with application to mitral valve mechanics. Constitutive modelling and finite element implementation. Int. J. Numer. Methods Eng. 71, 987-1008 (2007)

15. Prot, V., Haaverstad, R., Skallerud, B.: Finite element analysis of the mitral apparatus: annulus shape effect and chordal force distribution. Biomech. Model. Mechanobiol. 8, 43-55 (2008)

16. Wang, Q., Sun, W.: Finite element modeling of mitral valve dynamic deformation using patient-specific multi-slices computed tomography scans. Ann. Biomed. Eng. 41, 142-153 (2013)

17. Dal Pan, F., Donzella, G., Fucci, C., Schreiber, M.: Structural effects of an innovative surgical technique to repair heart valve defects. J. Biomech. 38(12), 2460-2471 (2005)

18. Votta, M., Maisano, F., Soncini, M., Redaelli, A., Montevecchi, F.M., Alfieri, O.: 3-D computational analysis of the stress distribution on the leaflets after edge-to-edge repair of mitral regurgitation. J. Heart Valve Dis. 11(6), 810-822 (2002)

19. Votta, E., Maisano, F., Bolling, S.F., Alfieri, O., Montevecchi, F.M., Redaelli, A.: The geoform diseasespecific annuloplasty system: a finite element study. Ann. Thorac. Surg. 84(1), 92-101 (2007) 
20. Mansi, T., Voigt, I., Georgescu, B., Zheng, X., Mengue, E.A., Hackl, M.: An integrated framework for finite-element modeling of mitral valve biomechanics from medical images: application to MitralClip intervention planning. Med. Image Anal. 16(7), 1330-1346 (2012)

21. Sturla, F., Redaelli, A., Puppini, G., Onorati, F., Faggian, G., Votta, E.: Functional and biomechanical effects of the edge-to-edge repair in the setting of mitral regurgitation: consolidated knowledge and novel tools to gain insight into its percutaneous implementation. Cardiovasc. Eng. Technol. 6(2), 117-140 (2015)

22. Kong, F., Pham, T., Martin, C., Elefteriades, J., McKay, R., Primiano, C., Sun, W.: Finite element analysis of annuloplasty and papillary muscle relocation on a patient-specific mitral regurgitation model. PLoS ONE 13(6), e0198331 (2018)

23. Kong, F., Caballero, A., McKay, R., Sun, W.: Finite element analysis of MitraClip procedure on a patientspecific model with functional mitral regurgitation. J. Biomech. 104, 109730 (2020)

24. Zhong, Q., Zeng, W., Huang, X., Malong, S., Yun, L.: Constitutive modeling and finite element analysis of myxomatous mitral leaflet tissue. J. Mech. Med. Biol. 14(3), 1450031 (2014)

25. Abaqus: Abaqus Analysis User's Guide. Dassault Systemes, vol. 4 (2014)

26. Jett, S.V., Hudson, L.T., Baumwart, R., Bohnstedt, B.N., Mir, A., Burkhart, H.M., Holzapfel, G.A., Wu, Y., Lee, C.H.: Integration of polarized spatial frequency domain imaging (pSFDI) with a biaxial mechanical testing system for quantification of load-dependent collagen architecture in soft collagenous tissues. Acta Biomater. 102, 149-168 (2020)

27. Lee, C., Zhang, W., Liao, J., Carruthers, C.A., Sacks, J.I., Sacks, M.S.: On the presence of affine fibril and fiber kinematics in the mitral valve anterior leaflet. Biophys. J. 108(8), 2074-2087 (2015)

28. Sturla, F., Onorati, F., Puppini, G., Pappalardo, O.A., Selmi, M., Votta, E., Faggian, G., Redaelli, A.: Dynamic and quantitative evaluation of degenerative mitral valve disease: a dedicated framework based on cardiac magnetic resonance imaging. J. Thorac. Dis. 9(4), 225-238 (2017)

29. Spencer, A.J.M.: Constitutive theory for strongly anisotropic solids. In: Spencer, A.J.M. (ed.) Continuum Theory of the Mechanics of Fibre-Reinforced Composites. International Centre for Mechanical Sciences (Courses and Lectures), pp. 1-32. Springer, Wien (1984)

30. Holzapfel, G.A., Gasser, T.C., Ogden, R.W.: A new constitutive framework for arterial wall mechanics and a comparative study of material models. J. Elast. 61, 1-48 (2000)

31. Gasser, T.C., Ogden, R.W., Holzapfel, G.A.: Hyperelastic modelling of arterial layers with distributed collagen fibre orientations. J. R. Soc. Interface 3(6), 15-35 (2006)

32. McEvoy, E., Holzapfel, G.A., McGarry, P.: Compressibility and anisotropy of the ventricular myocardium: experimental analysis and microstructural modeling. J. Biomech. Eng. 140(8), 1-10 (2018)

33. May-Newman, K., Yin, F.C.: A constitutive law for mitral valve tissue. J. Biomech. Eng. 120(1), 38-47 (1998)

34. Zuo, K., Pham, T., Li, K., Martin, C., He, Z., Sun, W.: Characterization of biomechanical properties of aged human and ovine mitral valve chordae tendineae. J. Mech. Behav. Biomed. Mater. 62, 607-618 (2016)

35. Kunzelman, K.S., Cochran, R.P., Verrier, E.D., Eberhart, R.C.: Anatomic basis for mitral valve modelling. J. Heart Valve Dis. 3(5), 491-496 (1994)

36. Cochran, R.P., Kunzelman, K.S., Chuong, C.J., Sacks, M.S., Eberhart, R.C.: Nondestructive analysis of mitral valve collagen fiber orientation ASAIO. Transactions 37(3), 447-448 (1991)

37. Rego, B.V., Khalighi, A.H., Drach, A., Lai, E.K., Pouch, A.M., Gorman, R.C., Gorman, J.H. 3rd, Sacks, M.S.: A noninvasive method for the determination of in vivo mitral valve leaflet strains. Int. J. Numer. Methods Biomed. Eng. 34(12), e3142 (2018)

38. Levick, J.: An Introduction to Cardiovascular Physiology, vol. 5. Hodder Arnold, London (2010)

39. Stevanella, M., Maffessanti, F., Conti, C.A., Votta, E., Arnoldi, A., Lombardi, M., Parodi, O., Caiani, E.G., Redaelli, A.: Mitral valve patient-specific finite element modeling from cardiac MRI: application to an annuloplasty procedure. Cardiovasc. Eng. Technol. 2, 66-76 (2011)

40. Prot, V., Skallerud, B., Sommer, G., Holzapfel, G.A.: On modelling and analysis of healthy and pathological human mitral valves: two case studies. J. Mech. Behav. Biomed. Mater. 3(2), 167-177 (2010)

41. Pham, T., Sun, W.: Material properties of aged human mitral valve leaflets. J. Biomed. Mater. Res. 102(8), 2692-2703 (2014)

42. Holzapfel, G.A.: Determination of material models for arterial walls from uniaxial extension tests and histological structure. J. Theor. Biol. 238(2), 290-302 (2006)

43. Sacks, M.S., Enomoto, Y., Graybill, J.R., Merryman, W.D., Zeeshan, A., Yoganathan, A.P., Levy, R.J., Gorman, R.C., Gorman, J.H.: In-vivo dynamic deformation of the mitral valve anterior leaflet. Ann. Thorac. Surg. 82(4), 1369-1377 (2006)

44. Carpentier, A., Adams, D.H., Filsoufi, F.: Carpentier's Reconstructive Valve Surgery: From Valve Analysis to Valve Reconstruction, vol. I. Saunders (Elsevier), Philadelphia (2010) 
45. Pham, T., Kong, F., Martin, C., Wang, Q., Primiano, C., McKay, R., Elefteriades, J., Sun, W.: Finite element analysis of patient-specific mitral valve with mitral regurgitation. Cardiovasc. Eng. Technol. 8, 3-16 (2017)

46. Bischoff, J.: Continuous versus discrete (invariant) representations of fibrous structure for modeling nonlinear anisotropic soft tissue behavior. Int. J. Non-Linear Mech. 2(41), 167-179 (2006)

47. Zhang, W., Ayoub, S., Liao, J., Sacks, M.S.: A meso-scale layer-specific structural constitutive model of the mitral heart valve leaflets. Acta Biomater. 32, 238-255 (2016)

48. Laurence, D., Ross, C., Jett, S., Johns, C., Echols, A., Baumwart, R., Towner, R., Liao, J., Bajona, P., $\mathrm{Wu}$, Y., Lee, C.H.: An investigation of regional variations in the biaxial mechanical properties and stress relaxation behaviors of porcine atrioventricular heart valve leaflets. J. Biomech. 83, 16-27 (2019)

49. Drach, A., Khalighi, A.H., Sacks, M.S.: A comprehensive pipeline for multi-resolution modeling of the mitral valve: validation, computational efficiency, and predictive capability. Int. J. Numer. Methods Eng. 34(2), 1-45 (2018)

50. Prot, V., Skallerud, B.: Contributions of prestrains, hyperelasticity, and muscle fiberactivation on mitral valve systolic performance. Int. J. Numer. Methods Eng. 33(4), 23014552 (2017)

51. Wit, A.L., Fenoglio, J.J., Hordof, A.J., Reemtsma, K.: Ultrastructure and transmembrane potentials of cardiac muscle in the human anterior mitral valve leaflet. Circulation 59(6), 1284-1292 (1979)

52. Filip, D.A., Radu, A., Simionescu, M.: Interstitial cells of the heart valves possess characteristics similar to smooth muscle cells. Circ. Res. 59(3), 310-320 (1986)

53. Sonnenblick, E.H., Napolitano, L.M., Daggett, W.M., Cooper, T.: An intrinsic neuromuscular basis for mitral valve motion in the dog. Circ. Res. 21(1), 9-15 (1967)

54. Curtis, M.B., Priola, D.V.: Mechanical properties of the canine mitral valve: effects of autonomic stimulation. Am. J. Physiol., Heart Circ. Physiol. 262(1), 56-62 (1992)

55. Montiel, M.M.: Muscular apparatus of the mitral valve in man and its involvement in left-sided cardiac hypertrophy. Am. J. Cardiol. 26(4), 341-344 (1970)

56. De Biasi, S., Vitellaro-Zuccarello, L., Blum, I.: Histochemical and ultrastructural study on the innervation of human and porcine atrio-ventricular valves. Anat. Embryol. 169(2), 159-165 (1984)

57. Kawano, H., Kawai, S., Shirai, T., Okada, R.: Morphological study on vagal innervation in human atrioventricular valves using histochemical method. Jpn. Circ. J. 57(8), 753-759 (1993)

58. Marron, K., Yacoub, M.H., Polak, J.M., Sheppard, M.N., Fagan, D., Whitehead, B.F., de Leval, M.R., Anderson, R.H., Wharton, J.: Innervation of human atrioventricular and arterial valves. Circulation 94(3), 368-375 (1996)

59. Timek, T.A., Lai, D.T., Dagum, P., Tibayan, F., Daughters, G.T., Liang, D., Berry, G.J., Miller, D.C., Ingels, N.B. Jr.: Ablation of mitral annular and leaflet muscle: effects on annular and leaflet dynamics. Am. J. Physiol., Heart Circ. Physiol. 285(4), 1668-1674 (2003)

60. Itoh, A., Krishnamurthy, G., Swanson, J.C., Ennis, D.B., Bothe, W., Kuhl, E., Karlsson, M., Davis, L.R., Miller, D.C., Ingels, N.B. Jr.: Active stiffening of mitral valve leaflets in the beating heart. Am. J. Physiol., Heart Circ. Physiol. 296(6), 1766-1773 (2009)

61. Nordrum, I.S., Skallerud, B.: Smooth muscle in the human mitral valve: extent and implications for dynamic modelling. APMIS, Acta Pathol. Microbiol. Immunol. Scand. 120(6), 484-494 (2012)

62. Amini, R., Eckert, C.E., Koomalsingh, K., McGarvey, J., Minakawa, M., Gorman, J.H., Gorman, R.C., Sacks, M.S.: On the in vivo deformation of the mitral valve anterior leaflet: effects of annular geometry and referential configuration. Ann. Biomed. Eng. 40(7), 1455-1467 (2012)

63. Rausch, M.K., Famaey, N., Shultz, T.O., Bothe, W., Miller, D.C., Kuhl, E.: Mechanics of the mitral valve: a critical review, an in vivo parameter identification, and the effect of prestrain. Biomech. Model. Mechanobiol. 12(5), 1053-1071 (2013)

64. Skallerud, B., Prot, V., Nordrum, I.S.: Modeling active muscle contraction in mitral valve leaflets during systole: a first approach. Biomech. Model. Mechanobiol. 10, 11-26 (2011)

Publisher's Note Springer Nature remains neutral with regard to jurisdictional claims in published maps and institutional affiliations. 